\title{
Structure and evolution of super-Earth to super-Jupiter exoplanets
}

\section{Heavy element enrichment in the interior}

\author{
I. Baraffe ${ }^{1}$, G. Chabrier ${ }^{1}$, and T. Barman ${ }^{2}$ \\ 1 École normale supérieure de Lyon, CRAL (CNRS), 46 allée d'Italie, 69007 Lyon; Université de Lyon, France \\ e-mail: [ibaraffe; chabrier]@ens-lyon.fr \\ ${ }^{2}$ Lowell observatory, 1400 West Mars Hill Road, Flagstaff, AZ 86001, USA \\ e-mail: barman@lowell.edu
}

Received 22 December 2007 / Accepted 11 February 2008

\begin{abstract}
Aims. We examine the uncertainties in current planetary models and quantify their impact on the planet cooling histories and massradius relationships.

Methods. These uncertainties include (i) the differences between the various equations of state used to characterize the heavy material thermodynamical properties, (ii) the distribution of heavy elements within planetary interiors, (iii) their chemical composition, and (iv) their thermal contribution to the planet evolution. Our models, which include a gaseous $\mathrm{H} / \mathrm{He}$ envelope, are compared with models of solid, gasless Earth-like planets in order to examine the impact of a gaseous envelope on the cooling and the resulting radius.

Results. We find that, for a fraction of heavy material larger than $20 \%$ of the planet mass, the distribution of the heavy elements in the planet's interior substantially affects the evolution and thus the radius at a given age. For planets with large core mass fractions ( $\gtrsim 50 \%$ ), such as the Neptune-mass transiting planet GJ 436b, the contribution of the gravitational and thermal energy from the core to the planet cooling history is not negligible, yielding a $\sim 10 \%$ effect on the radius after 1 Gyr. We show that the present mass and radius determinations of the massive planet Hat-P- $2 \mathrm{~b}$ require at least $200 M_{\oplus}$ of heavy material in the interior, at the edge of what is currently predicted by the core-accretion model for planet formation. As an alternative avenue for massive planet formation, we suggest that this planet, and similarly HD 17156b, may have formed from collisions between one or several other massive planets. This would explain these planets unusually high density and high eccentricity. We show that if planets as massive as $\sim 25 M_{\mathrm{J}}$ can form, as predicted by improved core-accretion models, deuterium is able to burn in the $\mathrm{H} / \mathrm{He}$ layers above the core, even for core masses as high as $\sim 100 M_{\oplus}$. Such a result highlights the confusion provided by a definition of a planet based on the deuterium-burning limit. Conclusions. We provide extensive grids of planetary evolution models from $10 M_{\oplus}$ to $10 M_{\text {Jup }}$, with various fractions of heavy elements. These models provide a reference for analyzing the transit discoveries expected from the CoRoT and Kepler missions and for inferring the internal composition of these objects.
\end{abstract}

Key words. stars: planetary systems - stars: individual: GJ 436 - stars: individual: Hat-P-2 - stars: individual: HD 149026

\section{Introduction}

The number of newly discovered exoplanets transiting their parent star keeps increasing continuously, revealing a remarkable diversity in mean densities for planet masses ranging from Neptune masses to several Jupiter masses. A large fraction of these transits exhibits a mean density significantly higher than that of an object essentially composed of gaseous $\mathrm{H} / \mathrm{He}$, such as brown dwarfs or stars, indicating a composition substantially enriched in heavy elements. The first compelling evidence for such a significant enrichment was provided by the discovery of a Saturn mass planet, HD 149026b, with such a small radius that $2 / 3$ of the planet's mass must be composed of elements heavier than $\mathrm{He}$ (Sato et al. 2005). Another remarkable discovery is the case of GJ $436 \mathrm{~b}, \mathrm{a} \sim 22 M_{\oplus}$ Neptune-like planet with a radius comparable to that of Uranus or Neptune (Gillon et al. 2007a). Such a radius implies an inner structure composed of more than $90 \%$ of heavy elements. That exoplanets can be substantially enriched in heavy material such as rock or ice ${ }^{1}$ is not

\footnotetext{
${ }^{1}$ Under usual planet formation conditions, the word "rocks" refers primarily to silicates (Mg-, Si- and O-rich compounds) whereas the term
}

a surprise, since this is a well known property of our own Solar System planets. Moreover, the presence of an icy/rocky core and of oversolar metallicity in the envelope is an expected consequence of the most widely accepted planet formation scenario, the so-called core-accretion model (Alibert et al. 2005a, and references therein).

Given this expectation, it becomes mandatory to take heavy element enrichment into account in planetary models devoted to the analysis and the identification of current and forthcoming observations of extra-solar planets. Many efforts are now devoted to the modeling of massive terrestrial planets, essentially composed of solid material (Valencia et al. 2006; Sotin et al. 2007; Seager et al. 2007) and jovian planets with $\mathrm{H} / \mathrm{He}$ envelope and a substantial metal enrichment (Baraffe et al. 2006; Guillot et al. 2006; Burrows et al. 2007; Fortney et al. 2007). Because of remaining uncertainties in the input physics describing the planetary structures, and of many unknown quantities such as

"ice" includes collectively $\mathrm{H}_{2} \mathrm{O}, \mathrm{CH}_{4}$ and $\mathrm{NH}_{3}$, water being the most important of these three components. As will be discussed in Sect. 3, the term "ice" may be inappropriate in some cases, as water could be under a liquid or gaseous form. 
the total amount of heavy elements, their chemical composition and their distribution within the planet's interior, these models are based on a number of assumptions and thus retain some, so far unquantified, uncertainties. The main goal of the present paper is to analyse and quantify these uncertainties and to explore as precisely as possible the impact of the heavy material contribution on the planet structure and evolution. We will focus on planets with mass larger than $10 M_{\oplus}$, the expected limit for the gravitational capture of a gaseous $\mathrm{H} / \mathrm{He}$ envelope and atmosphere (Mizuno 1980; Stevenson 1982; Rafikov 2006; Alibert et al. 2006), which has a significant impact on the evolution. Below this limit mass, the objects are essentially solid bodies with no or a teneous gaseous envelope and their mass-radius relationship has been studied recently by several authors (Valencia et al. 2006, 2007; Sotin et al. 2007; Seager et al. 2007). This study is motivated by the level of accuracy on planetary mass and radius measurements which is now reached with ground-based (HARPS, VLT) and space-based instruments (HST, SPITZER). Observations are expected to reach an unprecedented level of precision in the near-future with COROT, KEPLER, and on a longer term with GAIA. The latter project will measure distances and thus stellar radii with high precision, removing one of the main sources of uncertainty in planetary radius measurements. This race for precision is motivated by the possibility to infer with the best possible accuracy the inner composition of an exoplanet, with the aim to better understand planet formation and to identify the presence of astrobiologically important material such as liquid water. In this context, it is crucial to quantify the uncertainties in the structure and evolution planetary models used to analyse these observations. In Sects. 2 and 3, we examine the main input physics and assumptions used in structure and evolutionary models available in the literature. In Sect. 4, we analyse quantitatively the impact of these assumptions. We focus on specific cases such as HD 149026b and GJ 436b in Sect. 5 and on super Jupiter planets in Sect. 6. Our various planetary models, covering a wide mass range and including different levels of heavy element enrichments, are presented in Sect. 7. Discussion and perspectives follow in Sect. 8.

\section{Uncertainties and assumptions in the modelling of extra-solar planets}

\subsection{Distribution of heavy material within the planet}

According to a recent study devoted to the structure of our giant planets (Saumon \& Guillot 2004), Jupiter should have a total amount of heavy elements ranging from 8 to $39 M_{\oplus}$, i.e. a metal mass fraction $Z \sim 2.5 \%$ to $12 \%$, with a maximum core mass of $11 M_{\oplus}$ and a maximum envelope metal mass fraction $Z_{\text {env }}=12 \%$. For Saturn, the same study suggests a total mass of heavy elements ranging from 13 to $28 M_{\oplus}$, i.e. $Z \sim 13 \%$ to $29 \%$, with a maximum core mass of $22 M_{\oplus}$ and a maximum $Z_{\text {env }} \sim 8 \%$. These properties can be understood within the standard general framework of giant planet formation: as a core is growing in mass due to accretion of planetesimals, it reaches a critical mass around $\sim 6-10 M_{\oplus}$ above which gas accretion begins (Mizuno 1980; Stevenson 1982; Rafikov 2006). During this gas accretion phase, planetesimals are still accreted and are either destroyed in the gas envelope or are falling onto the core, leading to further increase of the core mass. The fate of these accreted planetesimals, disrupted in the envelope or accreted onto the dense core, depends on their size, an unknown parameter in current planet formation models, and on the envelope mass. This general picture thus predicts that planets should have a dense core of heavy material (rock, water/ice) of, at least, a few Earth masses and can show different levels of heavy element enrichment in their envelope, depending on the accretion history (amount of gas accreted, size of planetesimals, etc.). Current models based on the core-accretion scenario are able to match the core mass and the envelope metal enrichment derived for Jupiter and Saturn (Alibert et al. 2005b), but predict in some cases much larger heavy element enrichments in the envelope, depending on the planet's mass, with values as large as $Z_{\text {env }} \gtrsim 50 \%$ for Neptune-size planets $\left(\sim 10-20 M_{\oplus}\right.$ ) (Baraffe et al. 2006).

Despite this widely accepted picture, current extra-solar planet models often simply assume that all heavy elements are located in the central core and that the envelope is either metalfree, $Z_{\text {env }}=0$ (Burrows et al. 2007; Fortney et al. 2007), or has a solar metallicity ${ }^{2}, Z_{\text {env }}=Z_{\odot}$ (Guillot et al. 2006). This simplification is based on the assumption that whether the heavy elements are located in the core or in the envelope should not affect the planet's evolution. The validity of such an assumption, however, has never been examined, as will be done in the present paper.

\subsection{Thermal contribution to the EOS}

An other simplification found in planet modelling is the use of temperature-independent EOS, assuming that the heavy element material is at zero-temperature or at a uniform, low temperature (Seager et al. 2007; Fortney et al. 2007). This is certainly a good assumption when examining the structure of terrestrial-like planets, composed essentially of solid (rocky/icy) material (Valencia et al. 2006; Sotin et al. 2007). This assumption, however, is not necessarily valid for the evolution of more massive planets. For these objects, the thermal and gravitational energy contributions of the core to the planet's cooling history are usually ignored and it is important to examine the impact of such a simplification.

\subsection{Heat transport}

Finally, a conventional assumption in planet modelling is to assume that the interiors (at least the gaseous envelope) of giant planets are homogeneously mixed, due to the dominant and supposedly efficient transport mechanism provided by large-scale convection. Accordingly, the internal temperature gradient is given by the adiabatic gradient, since superadiabaticity is negligible. The validity of this assumption, however, has been debated for the interior of our own jovian planets for already some time (Stevenson 1985) and has been questioned again more recently in the context of transiting extra-solar planets (Chabrier \& Baraffe 2007). It should be kept in mind, however, that, even though the planetary internal heat flux can only be carried out by convection (Hubbard 1968), the assumption of large-scale, fully adiabatic convection in planetary interiors has never been proven to be correct and even slightly inefficient convection can have a major impact on the planet's structure and evolution (Chabrier \& Baraffe 2007).

\footnotetext{
2 Note also that different envelope helium mass fractions have been assumed, with $Y=0.25$ (Burrows et al. 2007) or $Y=0.28$ (Fortney et al. 2007).
} 


\section{Equations of state for heavy elements}

\subsection{The case of water}

As quickly mentioned in the introduction, the generic term "ice" may often be inappropriate to describe the thermodynamic state of water under planetary conditions. Indeed, depending on the temperatures prevailing in the parent protoplanetary nebula at the initial location of the planet embryo, water could possibly be initially under the form of solid ice, but it could also be under the form of liquid or vapor. If initially solid, it may also melt or vaporize under the conditions prevailing in the planet's interior, or may also dissociate under the form of ionic melts at high pressures and temperatures (Schwegler et al. 2001). In fact, the phase diagram of heavy elements under the pressure and temperature conditions characteristic of the considered planet interiors is largely unknown, as only part of this diagram is presently accessible to high-pressure experiments or computer numerical simulations. Water, for instance, the dominant component after $\mathrm{H}$ and $\mathrm{He}$, is known to exhibit a complex phase diagram with many stable or metastable (amorphous) phases and several triple and critical points, because of the high flexibility of the hydrogen bonding. The melting line of water at high pressure and high temperature has been probed experimentally up to $P=3.5 \times 10^{11}$ dyn cm $^{-2}(0.35$ Mbar $)$ and $T=1040 \mathrm{~K} . \mathrm{H}_{2} \mathrm{O}$ has been found to remain solid at larger pressures and temperatures, suggesting that the melting line increases (in $T(P)$ ) at higher temperatures and extends up to at least $T \gtrsim 1600 \mathrm{~K}$ (Lin et al. 2005). Depending on its extrapolation at higher temperatures, this line may intersect the internal profiles of some of our planet models, at least in the Super-Earth and Neptune mass range, so that both liquid and solid $\mathrm{H}_{2} \mathrm{O}$ might be present at some depth in the interiors of these objects, as predicted for oceanplanets (Selsis et al. 2007), while supercritical $\mathrm{H}_{2} \mathrm{O}$ is more likely to be liquid or gaseous in the hotter interiors of Saturn-like or larger planets. As mentioned above, water is also found from shock-wave experiments and first-principles calculations to dissociate into $\mathrm{H}_{3} \mathrm{O}^{+}+\mathrm{OH}^{-}$ion pairs above $\sim 2000 \mathrm{~K}$ at $0.3 \mathrm{Mbar}$ (Schwegler et al. 2001). In fact, the distinction between "gas", "ice" and "rock" may become meaningless under extreme conditions. In that case, what matters is the global amount of heavy elements. The term "ice", or "water", may thus more generically refer to the volatile forms of $\mathrm{O}, \mathrm{C}$ and $\mathrm{N}$, while "rock" refers primarily to silicates ( $\mathrm{Mg}$-, $\mathrm{Si}$ - and O-rich compounds) and "iron" refers to the rest (metal, oxide, sulfide or substituting for $\mathrm{Mg}$ in the silicates) (Stevenson 1985).

\subsection{EOS available in the literature}

For Earth-like planets, models can test a variety of complex heavy element compositions inspired by the knowledge of the structure of our own Earth (Valencia et al. 2006, 2007; Sotin et al. 2007). Moreover, the EOS of materials which may be found in planetary interiors (water, iron, dunite or olivine, etc.) are reasonably well determined at zero-temperature or at $300 \mathrm{~K}$ (see Seager et al. 2007). Unfortunately, for larger planets, the exploration of internal compositions is restricted to a few materials for which EOS are available and cover a large enough range of pressures and temperatures. The two most widely used EOS in this context (Saumon \& Guillot 2004; Baraffe et al. 2006; Fortney et al. 2007; Burrows et al. 2007) are ANEOS (Thompson \& Lauson 1972) and SESAME (Lyon \& Johnson 1992), which describe the thermodynamical properties of water, rocks (olivine or dunite, i.e. $\mathrm{Mg}_{2} \mathrm{SiO}_{4}$, in ANEOS; a mixture of silicates and other heavy elements called "drysand" in SESAME) and iron. Figure 1 shows a comparison between these two EOS for the three aforementioned materials for two temperatures, namely $T=300 \mathrm{~K}$ and $T=6000 \mathrm{~K}$. Comparison is also shown with the zero-temperature EOS presented in Seager et al. (2007) for water, perovskite $\left(\mathrm{MgSiO}_{3}\right)$ and iron. These authors use fits to experimental data at low pressure $(P \lessgtr 2 \mathrm{Mbar})$ and an improved Thomas-Fermi-Dirac theory at high pressure $(P \gtrsim 100 \mathrm{Mbar})$, where the contribution of the degenerate electron fluid becomes dominant. As stressed by these authors, the main difficulty is to bridge the gap in the pressure regime 2 Mbar $\lesssim P \lesssim 100$ Mbar $\left(2 \times 10^{12}-10^{14}\right.$ dyne $\left.\mathrm{cm}^{-2}\right)$, which is the most relevant for planetary interiors. Their model EOS represents some improvement upon ANEOS and SESAME, in particular for water, but is valid only at zero-temperature.

The presently used EOS models (ANEOS and SESAME), and thus the inferred planet internal structures, thus retain a significant degree of uncertainty in the experimentally unexplored high- $P$ and high- $T$ domains. In these regimes, both EOS models, as well as the one used by Seager et al. (2007), are based on interpolations between experimental data at low or moderate density/temperature and well-known asymptotic limits, in general the Thomas-Fermi or more accurate density-functional type models, in the very high density, fully ionized limit. One can only hope that these interpolations do not depart too much from reality, as might be the case, for instance, if first-order phase transitions, which imply density and entropy discontinuities, occur in the regions of interest. For the static properties, this assumption is probably reasonable at high temperatures, but might be more questionable near melting lines. When addressing the transport properties, like e.g. the thermal diffusivity or kinematic viscosity, the results in the interpolated regime are definitely more doubtful.

As shown in Fig. 1, at low (room) temperature, the various EOS agree reasonably well. For water, the agreement lies within less than $5 \%$ at $P=0.1$ Mbar and within less than $16 \%$ at $P=100$ Mbar. For "rocks", keeping in mind that this term refers to different compositions in the various EOS, the agreement is comparable, although the difference can reach $27 \%$ at $P=0.1$ Mbar. For iron, the three EOS agree well at low pressure but differences as large as $\sim 20 \%$ can occur at $P=100 \mathrm{Mbar}$ between SESAME and Seager et al. (2007). Such cool temperature conditions, however, are more relevant to Earth-like planets than to the ones explored in the present study.

Figure 1 also shows, for ANEOS and SESAME, the variation of $P(\rho)$ with temperature, for conditions more suitable to our planetary interior conditions. The thermal contributions predicted by these EOS are significant at $P \lesssim 1$ Mbar, with a $\sim 40-60 \%$ difference in $P(\rho)$ between the $T=300 \mathrm{~K}$ and $T=6000 \mathrm{~K}$ isotherms, for iron and water, respectively. The differences keep increasing significantly for $T>6000 \mathrm{~K}$. It is instructive to quantify the impact of these thermal contributions on the evolution of our planets, and to determine whether neglecting the temperature dependence of the EOS is consequential or not.

Whereas $P(\rho)$ is the relevant quantity for the structure, the relevant one for the evolution is the entropy. Figure 2 portrays the $P$ - and $T$-dependence of the entropy, for three isotherms and isobars, for water, for the ANEOS and SESAME EOS, under conditions relevant to the planets of present interest. The values for the H/He fluid (EOS of Saumon et al. 1995; hereafter SCVH EOS) are also displayed for comparison. We see that, whereas the EOS agree reasonably well under Jovian-planet conditions, as expected as they reach the asymptotic, high- $P$, high- $T$ regime 


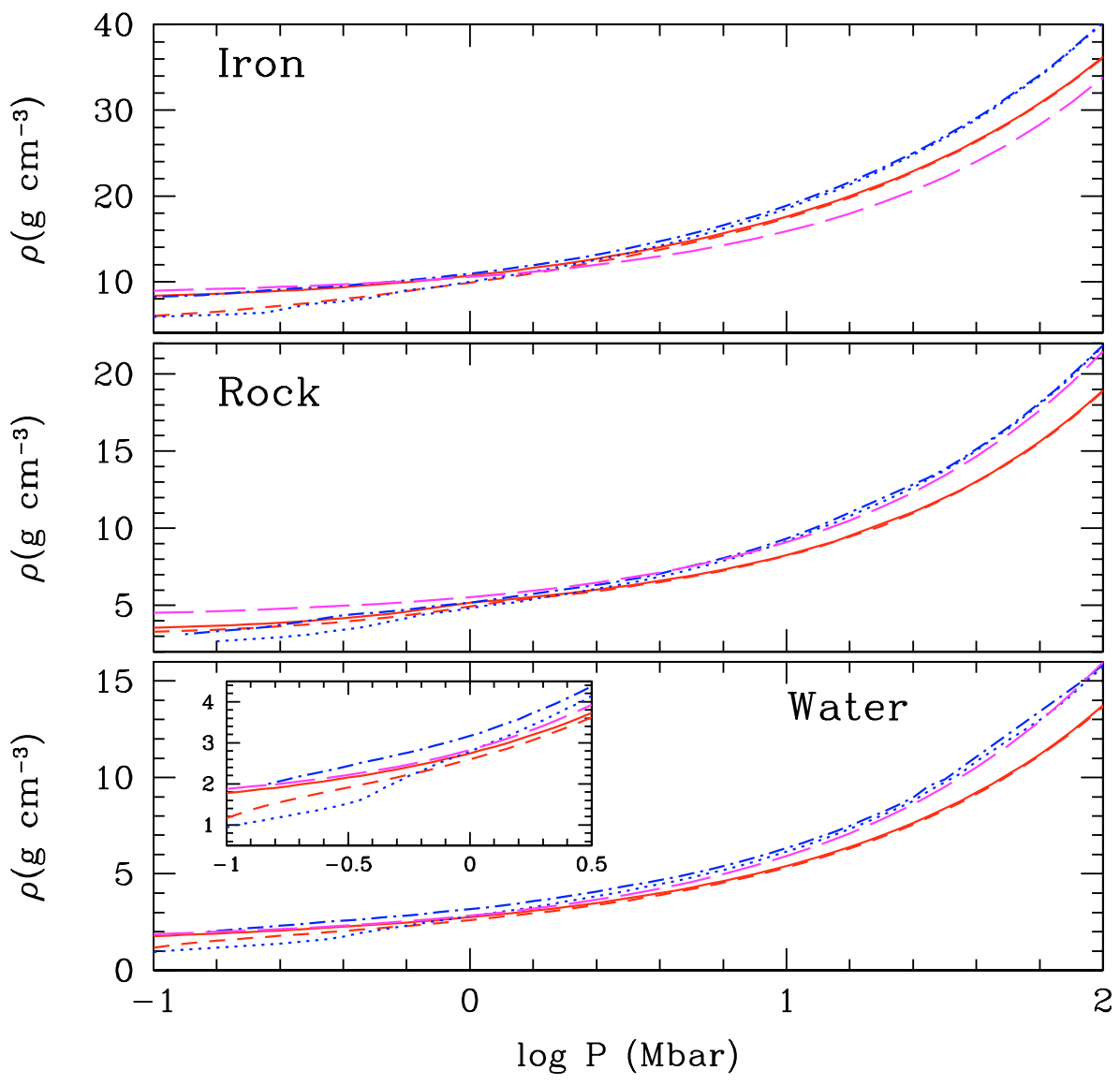

Fig. 1. EOS for water, rock and iron for two temperatures: $T=300 \mathrm{~K}$ for ANEOS (red solid line) and SESAME (blue dash-dotted curve); $T=6000 \mathrm{~K}$ for ANEOS (red short-dashed curve) and SESAME (blue dotted curve). The long-dashed (magenta) curves correspond to the zero-temperature EOS of Seager et al. (2007; their Table 3). The inset in the lower panel (water) shows a zoom of the $P(\rho)$ relation for $\log P<0.5$. accurately described by Thomas-Fermi-Dirac or more accurate density-functional theories, the difference can be substantial for conditions characteristic of the Neptune-mass domain. Indeed, for this latter case, most of the interior lies in the interpolated regime where guidance from either experiments or numerical simulations is lacking. These differences between the EOS, of course, are amplified for the quantities involving the derivatives of the entropy, such as the adiabatic gradient or the specific heat.

\subsection{Treatment of metal enrichment in the core and in the envelope}

Our goal is to examine the impact on the planetary models and the inferred mass-radius relationships due to uncertainties in (i) the distribution of heavy elements within the planet interiors, (ii) their dominant chemical composition, (iii) the different EOS describing their thermodynamical properties and (iv) their thermal contribution. To achieve this goal, we have implemented the ANEOS and SESAME EOS for water, rock and iron (or what is so-denominated) in our planetary evolution code. Both EOS provide all thermodynamic quantities relevant to the evolution of planets, including internal energy, entropy (in ANEOS) or free energy (in SESAME), and all relevant derivatives. ANEOS also provides Rosseland and conductive opacities. When the SESAME EOS is used, conductive opacities are calculated according to Potekhin (1999). The evolutionary calculations, including the presence of a dense core, proceed as described in Baraffe et al. (2006): the structure equations are integrated from the center to the surface; at the core boundary, the EOS is switched to the one characteristic of the gaseous envelope, and the change in chemical composition yields a density jump, but continuity in pressure and temperature is enforced.
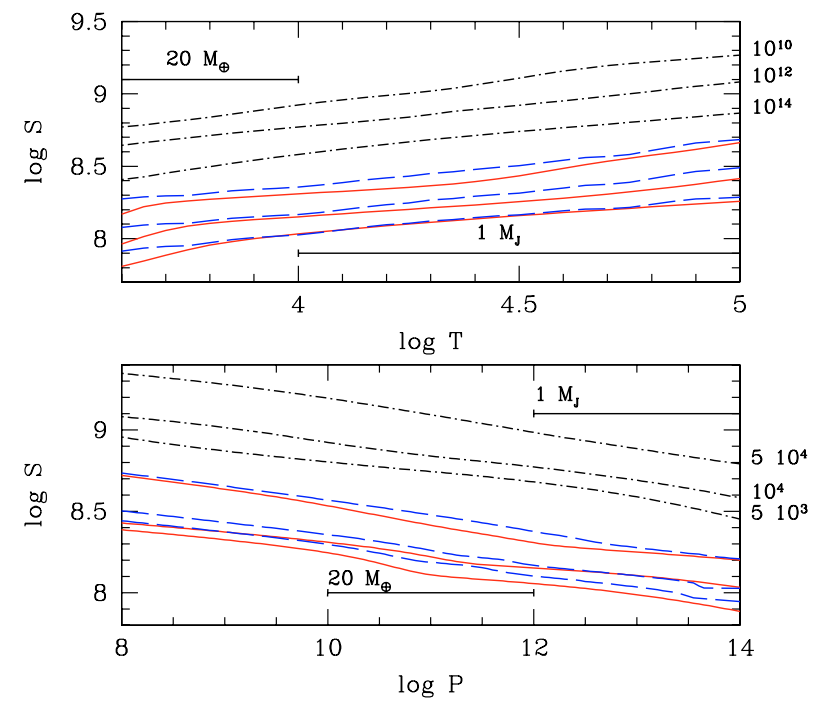

Fig. 2. Pressure (in dyne $\mathrm{cm}^{-2}$ ) and temperature (in $\mathrm{K}$ ) dependence of the entropy (in erg/g/s), as obtained for water for the ANEOS EOS (solid line) and the SESAME EOS (dash-line). The entropy for a $\mathrm{H} / \mathrm{He}$ fluid, with $Y=0.275$ obtained with the SCVH EOS is shown for comparison (dash-dotted line). Upper panel: for each EOS, three isobars are displayed (the corresponding pressure is given on the right hand side of the figure). Lower panel: three isotherms are shown for each EOS (corresponding temperatures given on the right hand side of the figure). The $T$ and $P$ domains characteristic of Neptune-like and Jupiter-like planet interiors are indicated.

To account for the thermodynamic contributions of heavy elements in the $\mathrm{H} / \mathrm{He}$ envelope, we have tested two different procedures. (1) Following the method described in 
Chabrier et al. (1992) and used in Baraffe et al. (2006), we first mimic the presence of metals with mass fraction $Z$ by an equivalent helium fraction $Y_{\text {equiv }}=Y+Z$ in the $\mathrm{H} / \mathrm{He} \mathrm{SCVH}$ EOS, with $Y$ the real helium mass fraction in the envelope. As mentioned in Chabrier et al. (1992), this approximation is reasonable as long as the mass fractions $Y$ and $Y_{\text {equiv }}$ are small compared with unity and both $\rho_{Z}$ and $\rho_{\mathrm{He}}$ are large compared with $\rho_{\mathrm{H}}$, where $\rho_{i}$ denotes the mass density of the $i$-component at pressure $P$. (2) The second, more general approach to describe the EOS of a mixture of various species in the absence of a reliable theory is to apply exactly the additive volume law (hereafter AVL), which is exact in the ideal gas limit, without restriction on the species mass fractions and densities (see Fontaine et al. 1977; and Saumon et al. 1995, for extensive discussions of the validity of the AVL). In this approach, the interactions between the three different fluids, namely hydrogen, helium and the heavy element component, are not taken into account (but interactions between particles in each of these fluids are treated properly) and the EOS of the mixture is simply the mass-weighted interpolation of each species contribution at constant intensive variables, $P$ and $T$, plus the ideal entropy of mixing for the entropy term. Within the ideal volume law, the mass density of the mixture of a $\mathrm{H} / \mathrm{He}$ fluid with a helium mass fraction $Y$ plus some heavy element material with mass fraction $Z$ at pressure $P$ and temperature $T$ thus reads:

$\frac{1}{\rho(P, T)}=\frac{(1-Z)}{\rho^{\mathrm{H} / \mathrm{He}}(P, T)}+\frac{Z}{\rho^{Z}(P, T)}$.

The extensive variables, e.g. the internal energy and specific entropy, thus read:

$$
\begin{aligned}
& U(P, T)=(1-Z) U^{\mathrm{H} / \mathrm{He}}(P, T)+Z U^{Z}(P, T) \\
& S(P, T)=(1-Z) S^{\mathrm{H} / \mathrm{He}}(P, T)+Z S^{Z}(P, T)+S_{\text {mix }}(P, T)
\end{aligned}
$$

where the EOS of the $\mathrm{H} / \mathrm{He}$ component is given by the SCVH EOS while the one of the Z-component is described by either the ANEOS or the SESAME EOS. All along the present calculations, we have taken the cosmic helium fraction $Y=0.275$. The details of the calculation of the contribution due to the ideal entropy of mixing are given in Appendix A. This term is found to contribute non negligibly to the total entropy $S$. Depending on the mixture and the $P-T$ range, it can amount to $10 \%-20 \%$ of $S$. This is consequential when calculating the internal structure of a planet, whose interior is essentially isentropic, at a given age.

For the evolution, the relevant quantities are the variation of the entropy with time and its derivatives w.r.t. $P$ and $T$, which give the adiabatic gradient:

$\nabla_{\mathrm{ad}}=\left(\frac{\partial \log S}{\partial \log P}\right)_{T} /\left(\frac{\partial \log S}{\partial \log T}\right)_{P}$.

Given the fact that the internal composition of the planet $\left(N_{\mathrm{H}}, N_{\mathrm{He}}, N_{Z}\right.$, where $N_{i}$ denotes the number of particles of species $i$ ), remains constant along the evolution, the only $P$-and $T$-dependence of the mixing entropy term arises from the variation of the degree of ionization and thus of the abundance of free electrons. The degree of ionisation of the heavy element component, however, is unknown, and its variation with temperature has been ignored in the present calculations. Therefore, only the variation with temperature of the number of electrons provided by $\mathrm{H}$ and $\mathrm{He}$ contribute to the variation with time of the mixing entropy. Within this limitation, the variation of $S_{\text {mix }}$ with $P$ and $T$ is found to be negligible for planets in the mass range of interest, for all levels of metal enrichment in the envelope, so that the entropy of mixing term does not contribute significantly to the evolution.

\section{Effect of the different treatments and distributions of heavy elements on the planet cooling history}

In this section, we analyse the impact of the localisation of the heavy elements within the planet on its structure and evolution. The heavy elements are distributed either in the core or in the gaseous envelope, and we consider in the present section mass fractions with $Z \leq 50 \%$. This encompasses the level of enrichment of our giant planets Jupiter and Saturn and of previous theoretical studies devoted to the analysis of transit planets (Guillot et al. 2006; Burrows et al. 2007). The effect of larger fractions of heavy elements $(Z>50 \%)$ will be explored in the next section, with the analysis of specific cases of planetary transits. To cover the largest possible range of conditions, we explore these effects on a Neptune mass $\left(20 M_{\oplus}\right)$ and a Jovian mass planet $\left(1 M_{\mathrm{J}}=318 M_{\oplus}\right)$. As a test case, we restrict the analysis in this section to planets irradiated by a Sun at $0.045 \mathrm{AU}$, since the observational determination of the radius is presently accessible only to transiting, short-period planets. We use outer boundary conditions derived from a grid of irradiated atmosphere models with solar metallicity (Barman et al. 2001). These models take into account the incident stellar flux, defined by $F_{\text {inc }}=\frac{f}{4}\left(\frac{R_{\star}}{a}\right)^{2} F_{\star}$, with $R_{\star}$ and $F_{\star}$ the radius and the flux of the parent star, respectively, and $f$ the redistribution factor. The Barman et al. (2001) models use $f=2$, corresponding to the incident flux being redistributed only over the dayside of the planet. Recent observations of the day-night contrast of exoplanets with Spitzer (see e.g. Knutson et al. 2007) and atmopsheric circulation models (see the discussion in Marley et al. 2007), however, seem to favor a redistribution over the entire planet's surface, i.e. $f=1$. For the specific case of HD209458b, Baraffe et al. (2003) found out that such a variation of $F_{\text {inc }}$ by a factor 2 has a significant effect on the outer atmospheric profile, but a small effect on the planet evolution. In a forthcoming paper, we will explore in more details the effects of the redistribution factor $f$, of different levels of irradiation and of heavy element enrichment in the atmosphere.

\subsection{Neptune-mass planets}

Figure 3 shows the evolution of the radius with time for a $20 M_{\oplus}$ planet with $Z=50 \%$, for different heavy element materials and for different distributions of these latter in the planet interior. We first consider models with a $10 M_{\oplus}$ core and $Z_{\text {env }}=0$, i.e. all heavy elements are located in the central core of the planet. The results for pure water (solid line), rock (dashed line) and iron (dash-dotted line) cores are displayed in Fig. 3a, based on the ANEOS EOS for these materials. A comparison of these results with those obtained with the SESAME EOS for the same material (water and drysand) shows less than a $1 \%$ difference on $\mathrm{R}$ at a given time. For iron, the SESAME EOS does not provide the free energy and thus the entropy, the adiabatic gradient and other quantities required for the evolution. However, according to the comparison between the ANEOS and SESAME EOS portrayed in Fig. 1, we do not expect significant differences on the evolution between the different iron EOS.

For the aforementioned global heavy element enrichment, $Z=50 \%$, the nature of the core material affects the cooling, and thus the radius evolution at the $\sim 10 \%$ level. We will see in Sect. 5, however, that for larger heavy element mass fractions, the impact of the core composition can be more severe. At $1 \mathrm{Gyr}$, the radius of the planet with a pure rocky (iron) core is 


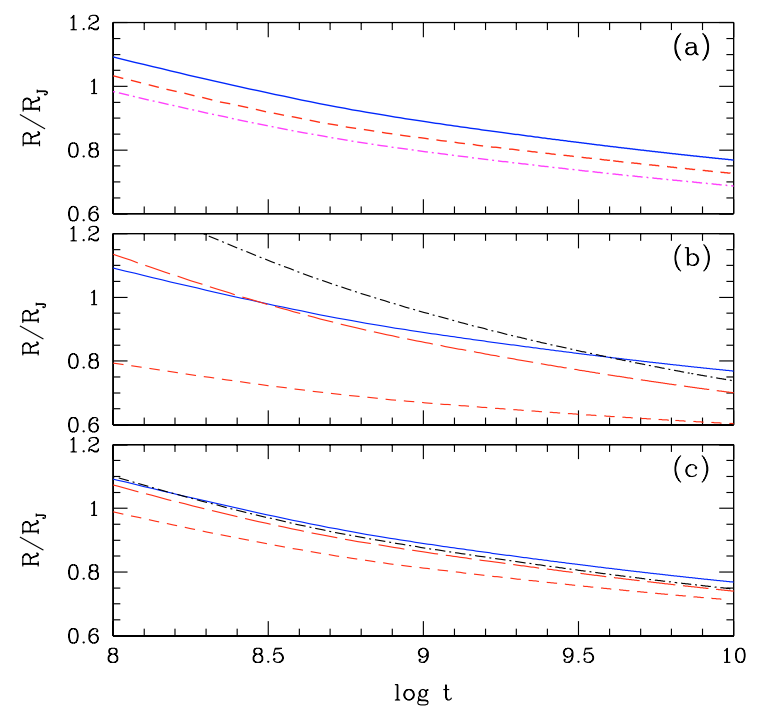

Fig. 3. Effect of the composition and internal distribution of heavy elements on the radius evolution for a planet of mass $M_{\mathrm{P}}=20 M_{\oplus}$ with a total heavy element mass fraction $Z=\left(M_{Z} / M_{\mathrm{P}}\right)=50 \%$. a) Models with $M_{\text {core }}=10 M_{\oplus}$ of water (solid line), rock (dashed-line) and iron (dotted line), and $Z_{\text {env }}=0$. b) Models with no core and heavy elements distributed over the entire planet. Dash-dotted line: mixture of $\mathrm{H} / \mathrm{He}+$ $Z$ described by the SCVH EOS with $Y_{\text {equiv }}=0.275+0.5=0.775$. Long-dashed line: mixture of $\mathrm{H} / \mathrm{He}+$ water (SESAME EOS) using the additive volume law. Short-dashed line: mixture of $\mathrm{H} / \mathrm{He}+$ water (ANEOS EOS) using the additive volume law. For comparison, the model with $M_{\text {core }}=10 M_{\oplus}$ of water and $Z_{\text {env }}=0$ is shown by the solid line. c) More realistic models with a $8 M_{\oplus}$ core of water (ANEOS EOS) and a $Z_{\mathrm{env}}=16.6 \%$ heavy element enrichment in the envelope. Dash-dotted line: mixture of $\mathrm{H} / \mathrm{He}+Z_{\text {env }}$ described by the SCVH EOS with $Y_{\text {equiv }}=0.275+0.166=0.441$. Long-dashed line: mixture of $\mathrm{H} / \mathrm{He}+$ water (SESAME EOS) using the additive volume law. Shortdashed line: mixture of $\mathrm{H} / \mathrm{He}+$ water (ANEOS EOS) using the additive volume law. Also displayed is the model with $M_{\text {core }}=10 M_{\oplus}$ of water and $Z_{\text {env }}=0$ (solid line).

smaller by $6 \%$ (11\%) compared to the pure water core case (see Table 1). For water and iron, the ANEOS EOS indicates whether the material is in a solid, liquid or melt phase. For dunite, this information is not provided. In all the models, water is always found to be in a liquid state. However, as mentioned in Sect. 3.1, most of our planet interiors lie in the extrapolated, supercritical region of the phase diagram; the precise state of water in this regime depends on the extrapolation of the melting curve and thus is presently undetermined. Moreover, as mentioned earlier, at high pressure and temperature, the meaning of "water" becomes loose and refers more generally to protons and oxygen nuclei. The planet with an iron core undergoes a phase change from liquid to solid, according to ANEOS, at an age of $\sim 1 \mathrm{Gyr}$. This transition starts in the most central part of the core, at temperatures $T \sim 1.7 \times 10^{4} \mathrm{~K}$ and $\rho \sim 30 \mathrm{~g} \mathrm{~cm}^{-3}$.

Figure $3 \mathrm{~b}$ illustrates the effect of the localisation of the heavy elements within the planet's interior, with two limiting assumptions: (A) all heavy elements are in the core $\left(Z_{\mathrm{env}}=0\right)$ and $(\mathrm{B})$ they are distributed all over the planet's interior $\left(M_{\text {core }}=0\right)$. In the latter case, we compare the two procedures mentioned in Sect. 3.2 to describe the thermodynamics properties of a mixture of $\mathrm{H} / \mathrm{He}$ and heavy elements. Comparison between these two limiting cases should provide the maximum effect on the planet's radius due to the unknown distribution of heavy elements within its interior, as long as large-scale adiabatic convection is considered as the heat transport mechanism. All the results portrayed in Fig. 3b are done with water. We find the following results:

- Under assumption (B), models based on a $Y_{\text {equiv }}$, for such a high metal fraction, $Z=50 \%$, lead to cooling sequences that differ drastically from the ones based on the AVL, with the SESAME EOS. This shows that the $Y_{\text {equiv }}$ simplification can not be used for such values of $Z$, as anticipated from the limitations of this assumption (see Sect. 3.3).

- Case (A) and case (B), with the AVL and the SESAME EOS, yield similar cooling sequences, with $\sim 4 \%$ difference at $t=$ 1 Gyr (7\% at 5 Gyr).

- In case B, we find a significant difference when using the AVL with ANEOS compared with all other cases ( $\sim 30 \%$ difference in $R$ for $t \geq 1$ Gyr compared with case (A), see Table 1). As mentioned in Sect. 3.2 and illustrated in Fig. 2, this stems from the different entropy dependence on $(P, T)$ predicted by the different EOS in the relevant pressure regime indicated in the middle panel of Fig. 4. Although all the evolution sequences are forced to start from the same initial entropy state, the sequence based on the ANEOS EOS shows a much stronger variation of the entropy with time. This stems from the stronger variation of $S$ with $P$ and $T$ predicted by this EOS, for the mass range characteristic of Neptune-like planets (see Fig. 2). This is highlighted in the upper panel of Fig. 4: the sequence based on the AVL with ANEOS shows $\sim 10$ times larger local gravothermal energy, $-T \mathrm{~d} S / \mathrm{d} t$, at the beginning of the evolution. Consequently, this sequence contracts and cools much faster than the other ones, loosing its internal entropy at a faster rate (lower panel of Fig. 4) and reaching a significantly smaller radius after 1 Gyr (see Fig. 3b).

The reason why the difference between the entropy, $S(P, T)$, obtained with the ANEOS and SESAME EOS (Figs. 2 and Fig. 3) is inconsequential when all the $Z$-material is located in the core, whereas it yields significantly different cooling sequences when the $Z$-component is mixed with the $\mathrm{H} / \mathrm{He}$ envelope stems from the fact that the evolution of a planet with a core is dominated by the entropy variation of the $\mathrm{H} /$ He envelope, $|\mathrm{d} S / \mathrm{d} t|_{\mathrm{HHe}} \gg|\mathrm{d} S / \mathrm{d} t|_{Z}$, a consequence of the smaller compressibility, $[\rho(\mathrm{d} P / \mathrm{d} \rho)]^{-1}$, and smaller specific heat of the $Z$-material compared with the $\mathrm{H} / \mathrm{He}$ one because of its much larger mean molecular weight (see Sect. 4.3).

Figure $3 \mathrm{c}$ also shows the evolution of a model with a more realistic distribution of the heavy material in the interior, namely a $8 M_{\oplus}$ core surrounded by a gaseous envelope with a $Z_{\mathrm{env}}=$ $16.6 \%$ heavy element mass fraction, which corresponds to a total heavy element enrichment of $50 \%$. First of all, we note that, for this value of $Z$, the cooling sequence base on the $Y_{\text {equiv }}$ formalism (dash-dot line) is very similar (within $\lesssim 2 \%$ ) to the one based on the more rigorous AVL (long-dash line). This shows that the $Y_{\text {equiv }}$ approximation for the treatment of a multispecies $\mathrm{H} / \mathrm{He} / Z$ EOS can be used relatively safely up to $Z \approx 20 \%$. Second of all, sequences with $M_{\text {core }}=8 M_{\oplus}, Z_{\text {env }}=16.6 \%$ (with the AVL and the SESAME EOS or with a $Y_{\text {equiv }}$ ) and the ones with $M_{\text {core }}=0$, $Z_{\text {env }}=50 \%$ (solid line) differ by less than $4 \%$. Therefore, for such levels of $Z_{\text {env }}$, the models are less sensitive to the treatment of the Z-element in the envelope. As mentioned previously, the sequence based on the ANEOS EOS (short-dash line) yields a significantly faster cooling sequence, with a $\sim 6 \%-10 \%$ smaller radius at $1 \mathrm{Gyr}$, compared with all other sequences. This large uncertainty on the EOS will be the major culprit for preventing accurate determinations of the exoplanet internal composition from their observed radius. 
Table 1. Radius and central thermodynamic properties of a planet of mass $M_{\mathrm{P}}=20 M_{\oplus}$ at $1 \mathrm{Gyr}$ with a total heavy element mass fraction $Z=$ $\left(M_{\mathrm{Z}} / M_{\mathrm{P}}\right)=50 \%$. Results for different heavy element compositions $($ water $=\mathrm{W}$, rock $=\mathrm{R}$, iron $=\mathrm{I})$ and $\mathrm{EOS}($ aneos $=\mathrm{a}, \mathrm{ses} a \mathrm{me}=\mathrm{s}, \mathrm{SCVH}=\mathrm{SC})$ and different heavy element distributions are given.

\begin{tabular}{cllllll}
\hline \hline $\begin{array}{c}M_{\text {core }} \\
\left(M_{\oplus}\right)\end{array}$ & EOS (core) & $Z_{\text {env }}$ & $\begin{array}{l}\text { EOS(env) } \\
\text { env. }\end{array}$ & $\begin{array}{l}R_{\mathrm{p}} \\
\left(R_{\mathrm{J}}\right)\end{array}$ & $\begin{array}{l}T_{\mathrm{c}} \\
(\mathrm{K})\end{array}$ & $\begin{array}{l}\rho_{\mathrm{c}} \\
\left(\mathrm{g} \mathrm{cm}^{-3}\right)\end{array}$ \\
\hline 10 & W-a & 0 & $\mathrm{SC}$ & 0.890 & $1.1 \times 10^{4}$ & 4.11 \\
10 & $\mathrm{~W}-\mathrm{s}$ & 0 & $\mathrm{SC}$ & 0.901 & $1.5 \times 10^{4}$ & 4.85 \\
10 & $\mathrm{R}-\mathrm{a}$ & 0 & $\mathrm{SC}$ & 0.836 & $8.1 \times 10^{3}$ & 9.07 \\
10 & $\mathrm{R}-\mathrm{s}$ & 0 & $\mathrm{SC}$ & 0.838 & $1.8 \times 10^{4}$ & 10.64 \\
10 & $\mathrm{I}-\mathrm{a}$ & 0 & $\mathrm{SC}$ & 0.799 & $1.7 \times 10^{4}$ & 31.20 \\
0 & & 0.5 & $\mathrm{SC}\left(0.775^{a}\right)$ & 0.953 & $1.2 \times 10^{4}$ & 0.58 \\
0 & & 0.5 & $\mathrm{SC}+\mathrm{W}-\mathrm{a}$ & 0.669 & $7.6 \times 10^{3}$ & 1.03 \\
0 & & 0.5 & $\mathrm{SC}+\mathrm{W}-\mathrm{s}$ & 0.858 & $10^{4}$ & 0.73 \\
8 & $\mathrm{~W}-\mathrm{a}$ & 0.166 & $\mathrm{SC}\left(0.441^{a}\right)$ & 0.876 & $1.2 \times 10^{4}$ & 3.85 \\
8 & $\mathrm{~W}-\mathrm{a}$ & 0.166 & $\mathrm{SC}+\mathrm{W}-\mathrm{a}$ & 0.811 & $1.1 \times 10^{4}$ & 3.93 \\
8 & $\mathrm{~W}-\mathrm{a}$ & 0.166 & $\mathrm{SC}+\mathrm{W}-\mathrm{s}$ & 0.862 & $1.1 \times 10^{4}$ & 3.87 \\
\hline
\end{tabular}

${ }^{a}$ Value of $Y_{\text {equiv }}$.

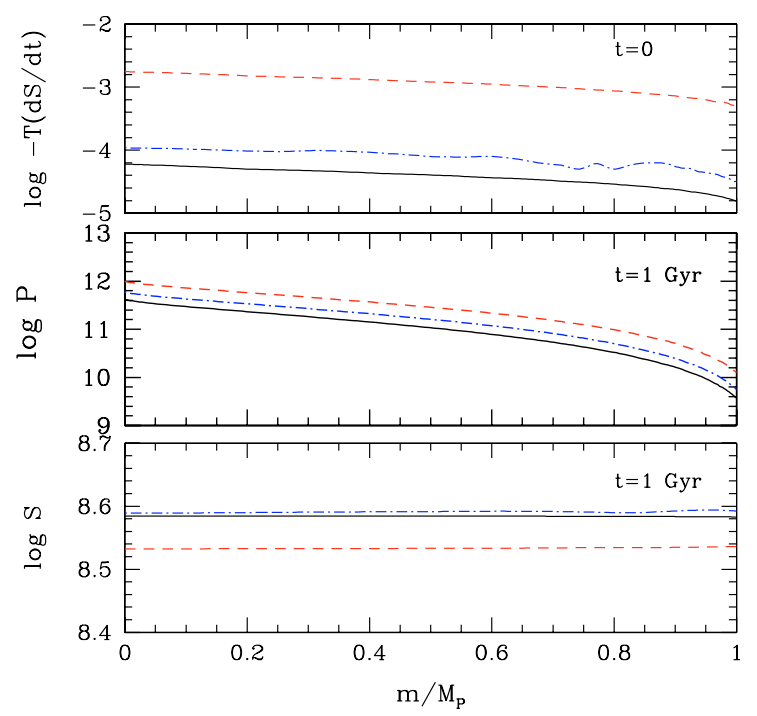

Fig. 4. Effect of the EOS on the inner profile as a function of fractional mass for a planet of $20 M_{\oplus}$ with a mass fraction $Z=50 \%$ of heavy elements distributed over the entire planet. In all panels, the curves correspond to: a mixture of $\mathrm{H} / \mathrm{He}+\mathrm{Z}$ described by: the $\mathrm{SCVH}$ EOS with $Y_{\text {equiv }}=0.275+0.5=0.775$ (solid line); a mixture of $\mathrm{H} / \mathrm{He}+$ water (SESAME EOS) using the additive volume law (dash-dotted line); a mixture of $\mathrm{H} / \mathrm{He}+$ water (ANEOS EOS) using the additive volume law (dashed line). Upper panel: inner profile of the local gravothermal energy, $-T \mathrm{~d} S / \mathrm{d} t$ (in erg/g/s), at the beginning of the evolution. Middle panel: pressure profile (in dyne $/ \mathrm{cm}^{2}$ ) at $1 \mathrm{Gyr}$. Lower panel: specific entropy profile (in $\mathrm{erg} / \mathrm{g} / \mathrm{K}$ ) at $1 \mathrm{Gyr}$.

We have also explored the effects of the heavy element distribution for smaller total enrichments, $Z<15 \%$. In this case, we find a $<2 \%$ global effect on the radius at a given age, depending whether the heavy elements are all located in the core or are distributed throughout the entire planet, using either an effective He abundance or the AVL with the SESAME EOS for the thermodynamics of the heavy component. Models based on the ANEOS EOS (with heavy elements distributed over the entire planet) still predict the smallest radii at a given time, with a maximum $\sim 10 \%$ effect compared with the other sequences.

Figure 5 portrays the internal $T(P)$ and $\rho(P)$ profiles for the various models of our $20 M_{\oplus}$ planet at an age of $4 \mathrm{Gyr}$.

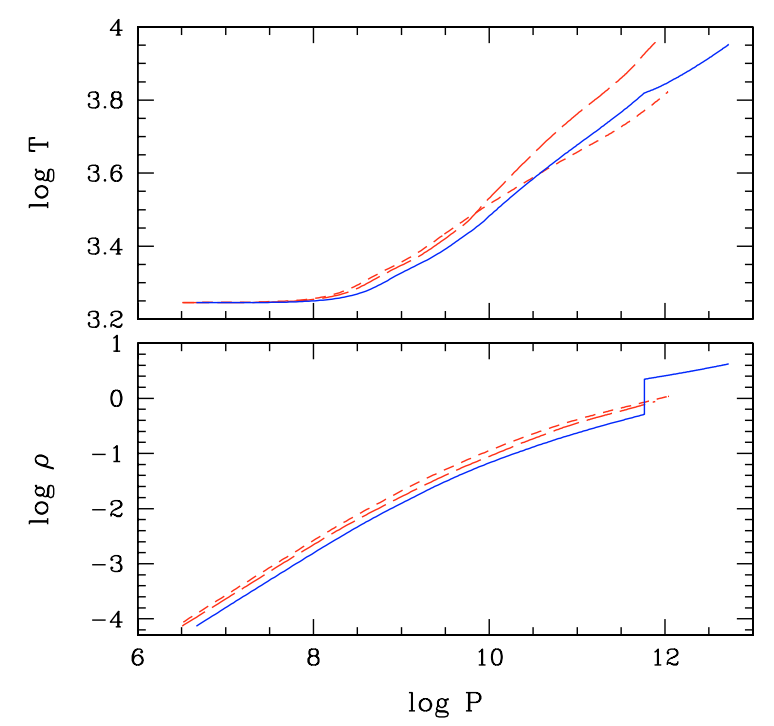

Fig. 5. Internal temperature $(\mathrm{K})$ and density $\left(\mathrm{g} \mathrm{cm}^{-3}\right)$ profiles for a $20 M_{\oplus}$ planet, irradiated by a Sun at $0.045 \mathrm{AU}$, at an age of $4 \mathrm{Gyr}$. Solid line: $M_{\text {core }}=0.5 M_{\mathrm{p}}, Z_{\text {env }}=0$; long-dash line: $M_{\text {core }}=0, Z=0.5$, AVL SESAME EOS; short-dash line: $M_{\text {core }}=0, Z=0.5$, AVL ANEOS EOS.

The first conclusions to be drawn from these tests for a Neptune-mass planet are the following:

(i) for a core mass less than $50 \%$ of the planet's mass, a variation of the core composition from pure water to pure iron, the maximum expected difference in mean densities, yields a difference on the radius of less than $\sim 10 \%$ after 1 Gyr. Yet, such a difference is accessible to the observational determination of some transit planet radii, if (when) other sources of uncertainties, in particular on the heavy material EOS, were (will be) under control;

(ii) for a metal-fraction in the envelope $Z_{\text {env }} \lesssim 20 \%$, the EOS of the $Z$-material can be approximated by using a corresponding $Y_{\text {equiv }}$ effective helium fraction in the SCVH EOS. This approximation become more dubious, and even wrong, above this limit;

(iii) if $Z \lesssim 15 \%$, either globally or in the envelope, the different treatments of heavy elements yield a relative variation of the radius of $\$ 2 \%$, except when using the ANEOS EOS, which yields $a \sim 10 \%$ difference. Within this limit for $Z$, and 
given all the other uncertainties in planet modelling, the impact of heavy elements on the evolution of Neptune-mass planets can be mimicked reasonably well by considering that the heavy elements are all located in the core;

(iv) for larger heavy element enrichments $(Z \gtrsim 15 \%)$, the distribution (everything in the core versus uniform distribution) and the treatment of the heavy element thermodynamic properties ( $Y_{\text {equiv }}$ or AVL) can significantly affect the cooling and thus radius determination for a given age (more than 10\% difference after $1 \mathrm{Gyr}$ ). These uncertainties, unfortunately, hamper an accurate determination of the detailed composition of the heavy element material in the planet's interior.

\subsection{Jovian-mass planets}

In this section, we extend the analysis done in the previous section to a template $1 M_{\mathrm{J}}$ planet. We first analyse the effect of core composition for a core mass of $159 M_{\oplus}$, corresponding to a total heavy element enrichment $Z=50 \%$ (see Fig. 6a). We find slightly larger effects than for the Neptune-mass case, with a $7 \%$ $(15 \%)$ difference in radius between the pure water and the pure rock (iron) core cases, respectively. Whether such massive cores can indeed form for Jovian-mass planets will be considered in Sect. 7.1. As for the Neptune case, using either the ANEOS or the SESAME EOS for the same material in that case yields similar cooling sequences, with $\lesssim 3 \%$ differences on $R$ for water and $\$ 2 \%$ for rock. For these planets, the iron core does not undergo a phase transition and iron always remains liquid, according to the ANEOS EOS. Indeed, central temperatures and densities are significantly larger than for Neptune-mass planets, with $T_{\mathrm{c}} \sim 10^{5} \mathrm{~K}$ and $\rho_{\mathrm{c}} \sim 157 \mathrm{~g} \mathrm{~cm}^{-3}$ at $1 \mathrm{Gyr}$ for a $1 M_{\mathrm{J}}$ planet with an iron core (see Table 2).

We explore the effects of the heavy element distribution with $Z=50 \%$ (Fig. 6b) and $Z=20 \%$ (Fig. 6c). The latter case is comparable to the expected level of enrichment in Jupiter and Saturn (Saumon \& Guillot 2004). In the $Z=50 \%$ case, both the various thermodynamic treatments and localizations of the heavy elements yield significantly different cooling behaviors (see Fig. 6b). The sequence based on the $Y_{\text {equiv }}$ approximation, notably, differs from the other ones, as expected from our previous study for Neptune-mass planets. At $1 \mathrm{Gyr}$, the radii obtained with models based on the AVL with ANEOS and SESAME, respectively, differ by $\sim 6 \%$. The different distributions of heavy elements within the planet have an even larger impact, with up to $12 \%$ difference in $R$ between the case with $M_{\text {core }}=159 M_{\oplus}$, $Z_{\text {env }}=0$ and the case with $Z$ distributed throughout the entire planet with ANEOS. Interestingly enough, for the present Jovian conditions, models based on the ANEOS or SESAME EOS, when metals are mixed throughout the entire planet, yield smaller differences than for the Neptune-mass planet case, after about $1 \mathrm{Gyr}$, i.e. at high pressure and moderately high temperature. Indeed, the inner pressure and temperature conditions are very different between Neptune-mass and Jupiter-mass planets (see Figs. 5 and 7). For the former ones, $P$ ranges between $10^{10}$ and $10^{12}$ dyne $^{-2}$ over more than $99 \%$ of the total mass (see Fig. 4) while $T$ ranges from $\sim 5000$ to $10^{4} \mathrm{~K}$. For the latter ones, the typical domains are $P=10^{12}-10^{14}$ dyne $^{-2}$ and $T=10^{4}-10^{5} \mathrm{~K}$. As mentioned in Sect. 3.2, differences in the $P$ and $T$ dependence of the entropy between the different EOS are more pronounced under the interior conditions of Neptune-mass planets than for Jupiter-mass planets (see Fig. 2).

If, instead of the extreme $M_{\text {core }}=0$ or $Z_{\text {env }}=0$ cases, we take a more realistic model with a $10 M_{\oplus}$ core, comparable to

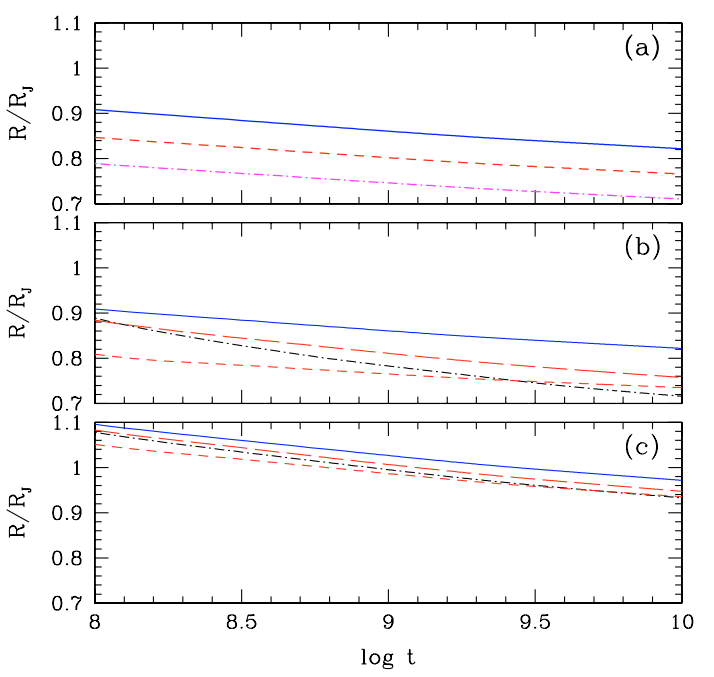

Fig. 6. Effect of the composition and the distribution of heavy elements on the radius evolution of a planet of $1 M_{\mathrm{J}}\left(318 M_{\oplus}\right)$. a) Total heavy element enrichment $Z=50 \%$. Models with $M_{\text {core }}=159 M_{\oplus}$ of water (solid line), rock (dashed-line) and iron (dotted line), and $Z_{\text {env }}=0$. b) Total heavy element enrichment $Z=50 \%$. The solid line corresponds to a model with $M_{\text {core }}=159 M_{\oplus}$ of water and $Z_{\text {env }}=0$. The other curves correspond to models with no core and heavy elements distributed over the entire planet: mixture of $\mathrm{H} / \mathrm{He}+\mathrm{Z}$ described by the SCVH EOS with $Y_{\text {equiv }}=0.275+0.5=0.775$ (dash-dotted line); mixture of $\mathrm{H} / \mathrm{He}+$ water (SESAME EOS) using the additive volume law (long-dashed line); mixture of $\mathrm{H} / \mathrm{He}+$ water (ANEOS EOS) using the additive volume law (short-dashed line). c) Total heavy element enrichment $Z=20 \%$. Solid line: model with $M_{\text {core }}=63.6 M_{\oplus}$ of water and $Z_{\text {env }}=0$; dash-dotted-line: no core and mixture of $\mathrm{H} / \mathrm{He}+Z$ described by the SCVH EOS with $Y_{\text {equiv }}=0.275+0.2=0.475$; long-dashed line: no core and mixture of $\mathrm{H} / \mathrm{He}+$ water (SESAME EOS) with the AVL and $Z_{\text {env }}=0.20$; short-dashed line: no core and mixture of $\mathrm{H} / \mathrm{He}+$ water (ANEOS EOS) using the AVL and $Z_{\text {env }}=0.20$.

what is expected in Jupiter or Saturn, and we distribute the rest of heavy elements homogeneously in the $\mathrm{H} / \mathrm{He}$ rich envelope, we find essentially the same evolution as when the heavy elements are distributed throughout the whole planet, with no core. This suggests that for massive, metal-rich planets, the evolution is better described by models which assume that all metals are distributed over the entire planet, since this yields results similar to the ones obtained with a more realistic distribution, than by models which assume that all heavy elements are in the core, with a metal-free, $Z=0$ envelope.

For a more moderate heavy element enrichment, $Z=20 \%$ (see Fig. 6c), the treatment of heavy elements in the entire planet, based on an $Y_{\text {equiv }}$ or on the AVL with ANEOS or SESAME, is found to be less consequential, for the present Jupiter-mass planet case. The different methods to describe the EOS yield less than $2 \%$ differences on $R$ at a given age. The effect of the distribution of heavy elements (all in the core versus all distributed over the entire planet) is slightly more consequential, with up to $4 \%$ difference on $R$.

Figure 7 portrays the internal $T(P)$ and $\rho(P)$ profiles for the various models of our $1 M_{\mathrm{J}}$ planet at an age of $4 \mathrm{Gyr}$.

The conclusions derived from these tests for a Jupiter-mass planet can be summarized as follows:

(i) for a core mass less than $50 \%$ of the planet's mass, a variation of the core composition between pure water and pure rock (iron) yields an effect on the radius of $\$ 7 \%(15 \%)$; 
Table 2. Radius and central thermodynamic properties of a planet of mass $M_{\mathrm{P}}=1 M_{\mathrm{J}}$ at $1 \mathrm{Gyr}$ for two heavy element mass fractions $Z=$ $\left(M_{Z} / M_{\mathrm{P}}\right)=50 \%$ and $Z=20 \%$. The labels are the same as in Table 1 . As mentioned at the end of Sect. 4.2 , for $Z=50 \%$, the solution with $M_{\text {core }}=0$ and $Z=Z_{\text {env }}$ is similar to a solution with $M_{\text {core }} \sim 10 M_{\oplus}$ and the rest of the heavy material distributed in the envelope.

\begin{tabular}{clllllll}
\hline \hline$Z$ & $\begin{array}{l}M_{\text {core }} \\
\left(M_{\oplus}\right)\end{array}$ & EOS (core) & $Z_{\text {env }}$ & $\begin{array}{l}\text { EOS(env) } \\
\text { env. }\end{array}$ & $\begin{array}{l}R_{\mathrm{p}} \\
\left(R_{\mathrm{J}}\right)\end{array}$ & $\begin{array}{l}T_{\mathrm{c}} \\
(\mathrm{K})\end{array}$ & $\begin{array}{l}\rho_{\mathrm{c}} \\
\left(\mathrm{g} \mathrm{cm}^{-3}\right)\end{array}$ \\
\hline 0.5 & 159 & $\mathrm{~W}-\mathrm{a}$ & 0 & $\mathrm{SC}$ & 0.861 & $5.3 \times 10^{4}$ & 20.89 \\
& 159 & $\mathrm{~W}-\mathrm{s}$ & 0 & $\mathrm{SC}$ & 0.841 & $6.8 \times 10^{4}$ & 28.31 \\
& 159 & $\mathrm{R}-\mathrm{a}$ & 0 & $\mathrm{SC}$ & 0.802 & $7.2 \times 10^{4}$ & 47.11 \\
& 159 & $\mathrm{R}-\mathrm{s}$ & 0 & $\mathrm{SC}$ & 0.789 & $8.6 \times 10^{4}$ & 59.87 \\
& 159 & $\mathrm{I}-\mathrm{a}$ & 0 & $\mathrm{SC}$ & 0.746 & $1.1 \times 10^{5}$ & 156.93 \\
& & 0.5 & $\mathrm{SC}\left(0.775^{a}\right)$ & 0.782 & $7.7 \times 10^{4}$ & 8.88 \\
& & & 0.5 & $\mathrm{SC}+\mathrm{W}-\mathrm{a}$ & 0.765 & $3.4 \times 10^{4}$ & 8.13 \\
& 0 & & 0.5 & $\mathrm{SC}+\mathrm{W}-\mathrm{s}$ & 0.811 & $5.8 \times 10^{4}$ & 7.36 \\
0.20 & & & & & & \\
& 63.6 & $\mathrm{~W}-\mathrm{a}$ & 0 & $\mathrm{SC}$ & 1.026 & $4.3 \times 10^{4}$ & 13.98 \\
& & & 0.2 & $\mathrm{SC}\left(0.475^{a}\right)$ & 0.994 & $3.9 \times 10^{4}$ & 4.38 \\
& & & 0.2 & $\mathrm{SC}+\mathrm{W}-\mathrm{a}$ & 0.986 & $3.2 \times 10^{4}$ & 4.18 \\
& & & 0.2 & $\mathrm{SC}+\mathrm{W}-\mathrm{s}$ & 1.006 & $3.7 \times 10^{4}$ & 4.06 \\
\hline
\end{tabular}

${ }^{a}$ Value of $Y_{\text {equiv }}$.
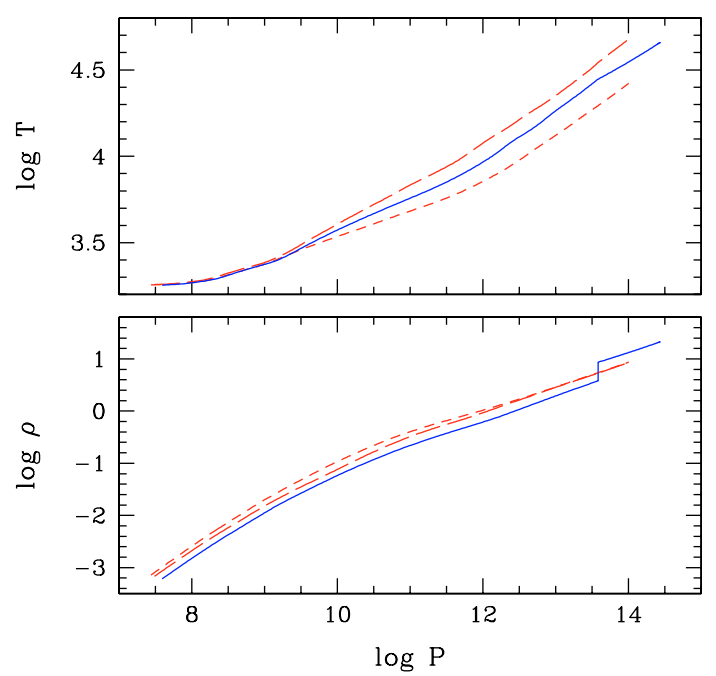

Fig. 7. Internal temperature and density profiles for a $1 M_{\mathrm{J}}$ planet at an age of 4 Gyr. Solid line: $M_{\text {core }}=0.5 M_{\mathrm{p}}$; long-dash line: $M_{\text {core }}=0$, $Z=0.5$, AVL SESAME EOS; short-dash line: $M_{\text {core }}=0, Z=0.5$, AVL ANEOS EOS.

(ii) for a global metal-enrichment $Z \lesssim 20 \%$, the treatment and distribution of heavy elements affect only modestly the radius predictions (less than $4 \%$ effect on $R$ at a given age);

(iii) for significant heavy element enrichment $(Z \sim 50 \%)$, the distribution of heavy elements (everything in the core versus uniform distribution) has a non negligible effect on the radius predictions, up to $12 \%$ difference at a given age. For such high metal-enrichments, distributing all the heavy material throughout the entire planet, with no core, is found to yield similar results as a more realistic model with about a $10 M_{\oplus}$ core and the rest of the material in the envelope, while a model with a metal-free envelope and all heavy elements in the core differs substantially from these sequences.

\subsection{Energy release from the core}

As mentioned in Sect. 2.2, the thermodynamics of the heavy material component in planet cores is often described either by a zero-temperature EOS or under the assumption of a uniform, low temperature (Fortney et al. 2007; Seager et al. 2007), or a uniform density (Bodenheimer et al. 2003). These models ignore the gravothermal energy contribution of the core, $\left(-T \frac{\mathrm{d} S}{\mathrm{~d} t}\right)_{\text {core }}$ to the cooling of the planet. As an other extreme, Burrows et al. (2007) arbitrarily assume that the specific entropy of the core material is the same as that of the $\mathrm{H} / \mathrm{He}$ envelope, therefore overestimating the heat release of the dense core.

According to the ANEOS or SESAME EOS, the typical heat capacity of water in the cores of the presently studied Neptune-mass and Jovian-mass planets is $C_{\mathrm{v}} \sim 3 \times 10^{7}-5 \times$ $10^{7} \mathrm{erg} \mathrm{g}^{-1} \mathrm{~K}^{-1}$, for typical temperatures $T \sim 5000-5 \times 10^{4} \mathrm{~K}$ and pressures $P \sim 10^{12}-10^{14}$ dyne $\mathrm{cm}^{-2}$ (see Figs. 5 and 7 ). This is about $1 / 3$ the specific heat of $\mathrm{H} / \mathrm{He}$ in the envelope. This corresponds to variations of the specific heat from the high-temperature nearly ideal gas limit, $\sim 6 \mathcal{R} / A$ (where $A$ is the species mean atomic weight and $\mathcal{R}$ is the perfect gas constant), to regimes which include potential-energy contributions associated with translational and librational modes $(3 \mathcal{R} / A$ maximum for three translational and three librational modes per molecule). If the species enters the solid phase, the specific heat decreases rapidly (Debye regime) and eventually vanishes. The specific heat of rock or iron is even smaller (larger atomic mass) and the contribution of cores made of such materials to the cooling history of planets is negligible. For planets in the mass range $20 M_{\oplus}-1 M_{\mathrm{J}}$, as explored in the previous section, with core masses less than $50 \%$ of the planet's mass, and with cores made up of water, the release of gravitational energy $\left(P \frac{\mathrm{d} V}{\mathrm{~d} t}\right)$ and of internal energy $\left(\frac{\mathrm{d} U}{\mathrm{~d} t}\right)$ never exceeds $40 \%$ of the total released energy, $T\left(\frac{\mathrm{d} S}{\mathrm{~d} t}\right)$. For Neptune-mass planets, the dominant contribution from the core is due to its contraction, i.e. the $P \frac{\mathrm{d} V}{\mathrm{~d} t}$ work, during the first $0.5-1$ Gyr of evolution, and by the release of its internal energy at older ages. For jovian-mass planets, the contraction of the core provides the dominant contribution to its gravothermal energy during the entire planet evolution.

The assumption of zero- $T$ or uniform low temperature affects the structure of the cores, because of the temperature dependence of the $P(\rho)$ relations predicted by current EOS (see Sect. 3.2 and Fig. 1). But, more importantly, it affects the evolution of the planet, because it implies a negligible contribution of the core to the total gravothermal energy of the planet. Indeed, the release of thermal energy from the core, $\left(\frac{\mathrm{d} U}{\mathrm{~d} t}\right)_{\text {core }}$, is forced to be zero, and the small compressibility of water at low $T$ 
drastically underestimates the true contraction of the core during the planet evolution, and thus the release of gravitational energy. We have analysed this assumption by imposing a constant and uniform temperature of $300 \mathrm{~K}$ in the water core of the planet models analysed in the previous section, using ANEOS and SESAME. For the jovian-mass planet $\left(1 M_{\mathrm{J}}\right)$ with $M_{\text {core }}=$ $159 M_{\oplus}$, the effect on the radius is negligible (less than $2 \%$ ) after 1 Gyr. On the Neptune-mass planet $\left(20 M_{\oplus}\right)$, the effect is larger, the largest effect being found with the SESAME EOS for the model with a $10 M_{\oplus}$ core, that yields a $6 \%$ effect on $R$ after 1 Gyr. The largest temperature variations of the EOS are indeed found at low pressure $(P<1$ Mbar $)$, as illustrated in Fig. 1, affecting more importantly light planets.

The heat transport in the core is due to convection or conduction (by electrons or phonons), depending on the core material and the age of the planet. Conduction may dominate after a few Gyr of evolution, as the core becomes cool and dense enough for the thermal conductivity due to degenerate electrons to become large enough, $K_{\mathrm{c}} \propto \rho^{4 / 3}$, and the conductive flux to dominate the convective one, $\mathcal{F}_{\text {cond }}=-K_{c} \nabla T>\mathcal{F}_{\text {conv }}$. It starts to dominate earlier for rock and iron cores, compared to water cases. These results, however, are hampered by the uncertainties in the conductive opacities calculated with the ANEOS EOS or with Potekhin (1999) for such materials. We have checked the effect of such an uncertainty on the cooling history in the case of the $20 M_{\oplus}$ planet with $M_{\text {core }}=10 M_{\oplus}$ of water with the SESAME EOS, as this sequence provides a case with the largest energetic contribution from the core, for enrichments $Z \leq 50 \%$. Over the entire evolution, heat is predicted to be transported by convection within the core and the temperature gradient is given by the adiabatic gradient. We have arbitrarily decreased the conductive opacities so that conduction now dominates over convection. The core thus becomes isothermal. The effect remains small on the radius evolution (maximum $3 \%$ on $R$ at a given age compared to the convective case). Therefore, the uncertainty in the heat transport efficiency of the core has a smaller impact than neglecting the temperature dependence of the core material and its global energetic contribution.

To conclude this section, the present calculations show that neglecting the thermal and gravitational contributions from the core, by assuming zero- $T$ or low uniform $T$, in current planet modelling, leads to a maximum effect of $\sim 6 \%$ on the radius, after 1 Gyr of evolution, for cores less massive than $50 \%$ of the planet's mass. Whether such a variation can be considered negligible or not depends on the accuracy of the data the models need to be confronted to in order to infer the planet's internal composition, once the EOS of heavy elements will be determine with enough accuracy. As will be shown in the next section, the conclusion is drastically different for larger metal contents, for Neptune-size planets.

\section{Extreme metal-enrichment: the two specific cases of HD 149026b and GJ 436b}

In this section, we analyse extreme cases of heavy element enrichments $(Z>50 \%)$, focusing on two recently discovered transiting planets, the Saturn-mass planet HD 149026b and the Neptune-mass planet GJ 436b. Our goal is to examine the properties of these two transit planets, in the light of the analysis conducted in the previous section concerning the main assumptions and uncertainties of current planetary models.

\section{1. $H D 149026 b$}

The discovery of the Saturn-mass planet HD 149026b (Sato et al. 2005) revealed an unexpectedly dense planet, with a mass $M_{\mathrm{P}}=0.36 \pm 0.04 M_{\mathrm{J}}$ and a radius $R_{\mathrm{P}}=0.725 \pm 0.05 R_{\mathrm{J}}$, i.e. a mean density $\bar{\rho}=1.17 \pm 0.35 \mathrm{~g} \mathrm{~cm}^{-3}$. For comparison, Saturn has a mass of $0.3 M_{\mathrm{J}}$ but a radius of $\sim 0.8 R_{\mathrm{J}}$, i.e. $\bar{\rho}=0.66 \mathrm{~g} \mathrm{~cm}^{-3}$. The transit planet is orbiting a G-type star at an orbital distance $a=0.042 \mathrm{AU}$, about 230 times closer to its Sun than Saturn. The age of the system, $\sim(2 \pm 0.8) \mathrm{Gyr}$, is also younger than our Solar System. It is important to understand the nature and the origin of this puzzling planet, and in particular to know whether current planet formation scenarios, in particular the core accretion model, can explain it. This requires a knowledge of its structure and composition, which can only be inferred from theoretical models. Several authors have tried to infer the inner structure of this planet under the usual assumptions described in Sect. 2. The models assume that almost all heavy elements are in the core, and the $\mathrm{H} / \mathrm{He}$ envelope is either free of metals (Burrows et al. 2007), or is moderately enriched, with $Z=Z_{\odot}$ (Ikoma et al. 2006) or $Z=0.045$ (Fortney et al. 2007). Ikoma et al. (2006) have also investigated a case with $Z_{\text {env }}=0.37$. Fortney et al. (2007) use a zero-temperature EOS for the core and thus ignore its heat content contribution. They find negligible effect when using a nonzero temperature EOS for the ice mixture given by Hubbard \& Marley (1989). This latter EOS provides pressuredensity relations appropriate for the description of warm adiabatic mixtures $\left(T \sim 10^{4}\right) \mathrm{K}$, but do not explicitly account for the temperature dependence. Ikoma et al. (2006) also use this EOS and assume a uniform temperature in the core and a specific heat $C_{\mathrm{v}} \sim 10^{7} \mathrm{erg} \mathrm{g}^{-1} \mathrm{~K}^{-1}$ to account for the core heat release. According to all these models, the total mass of heavy elements in the planet lies in the range $\sim 40-90 M_{\oplus}$, i.e. $Z \sim 35 \%-80 \%$.

We will test the impact of these main assumptions done in current structure models of HD 149026b, namely the distribution of all heavy elements in a core and the use of zero-T EOS. Since the present study focusses on the sensitivity of the structure and evolution for a given setup of atmosphere models, we use, as outer boundary condition to the inner structure, atmosphere models with solar composition, $Z_{\odot}$, irradiated by a G-star at $0.04 \mathrm{AU}$, even though the results are expected to change to some extent with the composition of the atmosphere (see e.g. Burrows et al. 2007). This issue will be explored in a forthcoming paper. We obtain a good match of the planet's radius with a model with a water core of $80 M_{\oplus}$ and a metal-free envelope, $Z_{\text {env }}=0$. Figure 8 displays this model (solid line), calculated with the ANEOS EOS in the core. The use of the SESAME EOS for water in the core yields a $3 \%$ smaller radius, still providing a good match to the observed value. Figure 8 also shows the impact of the heavy element distribution, with models where the $80 M_{\oplus}$ of heavy elements are distributed over the entire planet. As anticipated from the studies conducted in Sect. 3.2, the use of an $Y_{\text {equiv }}$ in the SCVH EOS to handle the heavy element contribution yields, for such a high metal fraction, an evolution that differs drastically from the other ones, even though, coincidentally, it gives a good solution at the age of the system. As expected from the tests performed in Sect. 4, models with $80 M_{\oplus}$ of heavy elements mixed with $\mathrm{H} / \mathrm{He}$ throughout the entire planet, using the AVL with the SESAME (long-dash line) or the ANEOS (short-dash line) EOS for water, yield denser structures. The latter one yields a radius $\sim 25 \%$ smaller than the observed value, an effect larger than changing the core composition from water to rock ( $\sim 10 \%$ effect on $R$ ), for the same core mass 


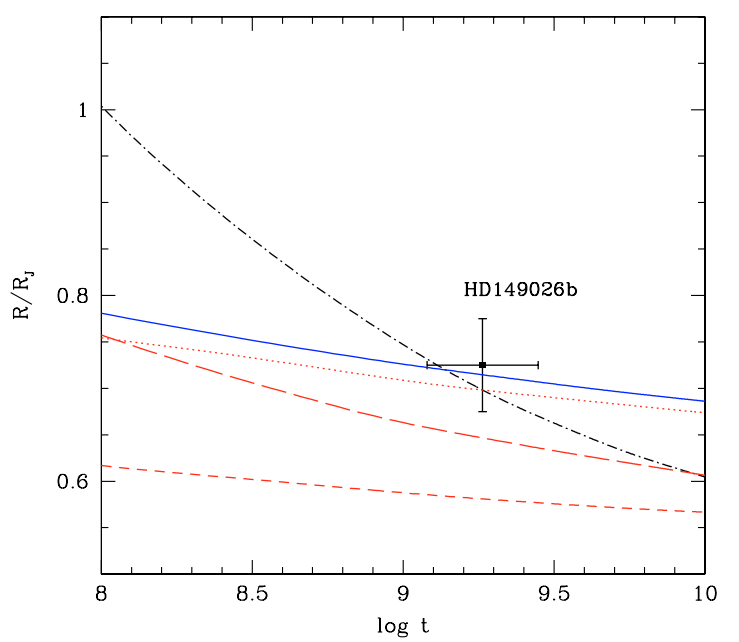

Fig. 8. Evolution of a planet with the characteristics of HD $149206 \mathrm{~b}$ $\left(m_{\mathrm{P}}=116 M_{\oplus} \sim 0.36 M_{\mathrm{J}}\right)$ with different heavy element distributions. Solid line: model with $M_{\text {core }}=80 M_{\oplus}$ of water (ANEOS EOS) and $Z_{\text {env }}=0$. The other curves correspond to models with heavy elements distributed over the entire planet. Dash-dot line: mixture of $\mathrm{H} / \mathrm{He}+$ $Z$ described by the SCVH EOS with an effective helium abundance, $Y_{\text {equiv }}=0.275+0.69=0.965$, which corresponds to $Z=0.69$, i.e. $80 M_{\oplus}$ of heavy elements. Long-dash line: mixture of $\mathrm{H} / \mathrm{He}+$ water (SESAME EOS) using the AVL with $Z=69 \%$. Short-dash line: mixture of $\mathrm{H} / \mathrm{He}+$ water (ANEOS EOS) using the AVL with $Z=69 \%$. Dotted line: mixture of $\mathrm{H} / \mathrm{He}+$ water (ANEOS EOS) using the AVL with $Z=51 \%$, i.e. $60 M_{\oplus}$ of heavy elements.

of $80 M_{\oplus}$, and similar to the one obtained when changing from a pure water to a pure iron core, a rather unlikely solution.

Instead of the two aforedescribed extreme heavy element distributions, we have also examined a more realistic model with a $20 M_{\oplus}$ core, similar to what is predicted for Saturn, and the remaining $60 M_{\oplus}$ mixed within the $\mathrm{H} / \mathrm{He}$ envelope, using the AVL both with SESAME and with ANEOS. As anticipated from the tests performed in Sect. 4.2, this yields similar results than assuming that all heavy elements are distributed throughout the entire planet. Models with such metal enrichment and distributions thus seem to be excluded by the observations. An alternative solution for HD 149026b, however, is obtained with a $60 M_{\oplus}$ of heavy elements, instead of 80 , mixed with $\mathrm{H} / \mathrm{He}$ throughout the entire planet, described with the ANEOS EOS for water and the AVL formalism (dotted line in Fig. 8), whereas when using the SESAME EOS for water, $70 M_{\oplus}$ are required. As mentioned above and as shown in our previous studies, such models are equivalent to models with a small core mass and a significantly enriched envelope. This shows that for a given heavy element material, water in the present case, the effect of modifying its internal distribution (everything in the core or a fraction redistributed in the $\mathrm{H} / \mathrm{He}$ envelope) has by itself a large impact, yielding an uncertainty on the amount of heavy material required to reproduce the observed radius of $80-60=20 M_{\oplus}$. As shown in the previous sections, assuming all heavy elements to be in the core yields structures less dense than when these elements are mixed throughout the envelope, so that these models require a larger amount of heavy material to match a given radius.

Finally, as done in Sect. 4.3, we have tested the effect of the temperature dependence of the EOS in the core by assuming a uniform temperature of $300 \mathrm{~K}$ in a model with a $80 M_{\oplus}$ water core, using ANEOS and SESAME EOS. The largest effect is found with this latter EOS, the model with a uniform low

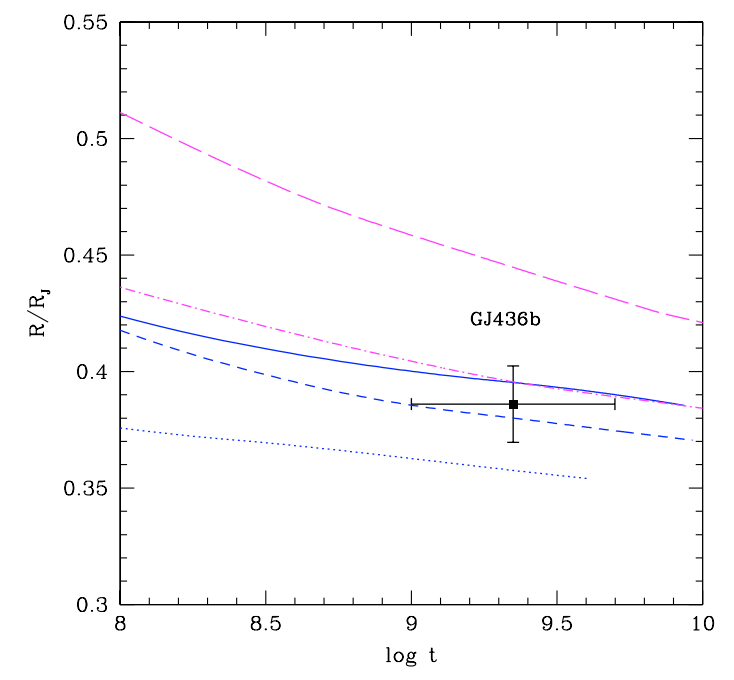

Fig. 9. Evolution of a planet characteristic of GJ 436b $\left(M_{\mathrm{P}}=22.6 M_{\oplus},\right)$ with different heavy element distributions. Models with no irradiation: Solid line: model with $M_{\text {core }}=21 M_{\oplus}$ of water (ANEOS EOS) and $Z_{\text {env }}=0$; short-dash line: model with $M_{\text {core }}=21 M_{\oplus}$ of water (SESAME EOS) and $Z_{\mathrm{env}}=0$; dotted line: test model with $M_{\text {core }}=$ $21 M_{\oplus}$ of water (SESAME EOS) and $Z_{\mathrm{env}}=0$, assuming an uniform temperature of $300 \mathrm{~K}$ in the core. Effect of irradiation, with $F_{\text {inc }}=6 \times F_{\text {inc }}$ for GJ $436 \mathrm{~b}$ (see text): long-dash line: same as short-dash line with irradiated atmosphere models; dash-dot line: same as dotted line with irradiated atmosphere models.

temperature yielding a radius $\sim 3 \%$ smaller than the "hot" core case at 2 Gyr.

\subsection{GJ $436 b$}

The first Neptune-mass transiting planet has been discovered recently (Gillon et al. 2007a), with a mass $M_{\mathrm{P}}=22.6 \pm 1.9 M_{\oplus}$ and a radius $R_{\mathrm{P}}=25200 \pm 2200 \mathrm{~km}=0.352 \pm 0.03 R_{\mathrm{J}}$. The planet is orbiting an M-type star of $\sim 0.44 M_{\odot}$ at an orbital distance $a \sim 0.028 \mathrm{AU}$. According to the models of Fortney et al. (2007), Gillon et al. (2007a) suggest that the planet is composed predominantly of ice with a thin $\mathrm{H} / \mathrm{He}$ envelope of less than $10 \%$ in mass. Based on Spitzer observations, Gillon et al. (2007b) and Deming et al. (2007) determine a slightly larger radius, with $R_{\mathrm{P}}=0.386 \pm 0.016 R_{\mathrm{J}}$. We adopt this value in the following and we assume an age for the system of 1-5 Gyr, as it is essentially unconstrained by the observations. Models available in the literature to determine the inner structure of this planet use temperature-independent EOS to describe the core and thus ignore its thermal contribution (Fortney et al. 2007; Seager et al. 2007; Adams et al. 2007). We will test this assumption.

We have calculated models characteristic of GJ 436b. We use solar metallicity atmosphere models and, given the low luminosity of the parent star, we neglect presently the irradiation effects. Recent determinations of GJ 436b irradiation induced temperature (i.e. brightness temperature) indeed suggest that the evolution is not likely to be significantly altered by irradiation (Demory et al. 2007). A model with a core of $21 M_{\oplus}$ made of water and a metal-free envelope, $Z_{\mathrm{env}}=0$, provides a good match to the observed radius, as illustrated in Fig. 9 (solid and dashed lines). The ANEOS EOS for water (solid line) yields a slightly larger $(\sim 4 \%)$ radius than the SESAME EOS (dashed line). A good match is also obtained with a rocky (dunite) core of mass $19.5 M_{\oplus}$. Given the high mass fraction of heavy material of this planet, more than $80 \%$, the freedom to vary its distribution is 


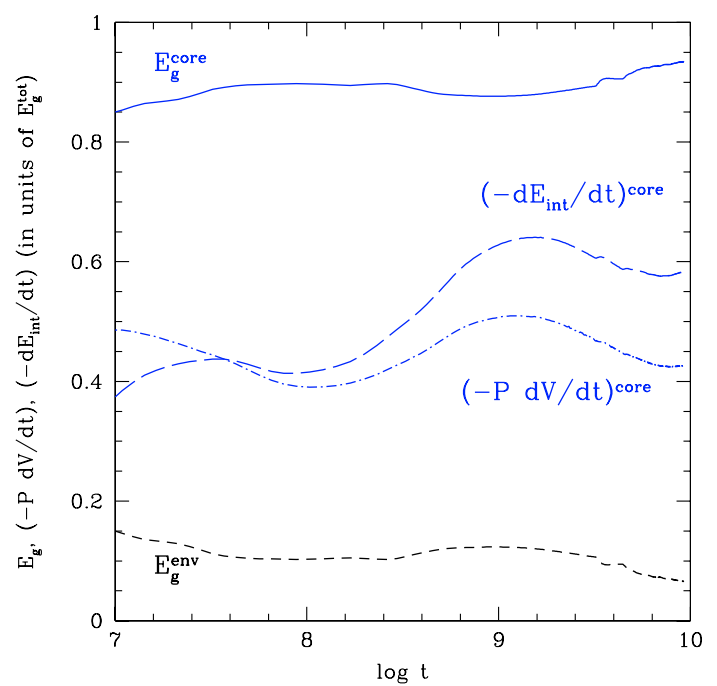

Fig. 10. Various contributions to the gravothermal energy, $E_{\mathrm{g}}=$ $-T \mathrm{~d} S / \mathrm{d} t$, normalized to the total value, for a planet characteristic of GJ $436 \mathrm{~b}\left(M_{\mathrm{P}}=22.6 M_{\oplus}\right)$, with a $21 M_{\oplus}$ core of water with the SESAME EOS. Solid line: global contribution from the core; dash-dot line: contraction work contribution from the core; long-dash line: thermal contribution from the core; short-dash line: global contribution from the $\mathrm{H} / \mathrm{He} 1.6 M_{\oplus}$ envelope.

limited. Assuming a core of $21 M_{\oplus}$ with $Z_{\text {env }}=0$ or a slightly smaller core with some heavy element enrichment in the envelope, a more realistic solution, e.g. a model with $M_{\text {core }}=20 M_{\oplus}$ and $Z_{\text {env }}=0.38$, yield less than $8 \%$ variations on the radius, still within the observed error bars. According to the tests performed in Sect. 4.1 on a $20 M_{\oplus}$ planet, larger values of $Z_{\text {env }}$ ( $Z_{\text {env }} \gtrsim 50 \%$ ), and thus smaller cores, yield larger effects on the radius. Note that, according to present planet formation models (Alibert et al. 2005a; Mordasini et al. 2008), such high fractions of $Z$-material enrichment in envelopes of Neptune-mass planets are not excluded (Baraffe et al. 2006, Fig. 1) since, as mentioned in Sect. 2.1, the fate of accreted planetesimals during the gas accretion phase depends on the envelope mass.

Figure 9 also displays the evolution of the aforementioned model with the assumption of a uniform cold temperature $(300 \mathrm{~K})$ in the core. This yields a moderate effect on the evolution, about $4 \%$ on the radius at $1-5 \mathrm{Gyr}$ with the ANEOS EOS and $\sim 6 \%$ effect with SESAME. In the latter case, however, the model lies outside the observational error bars (see dotted line in Fig. 9). As seen in Fig. 10, for planets with such a large fraction of heavy material, and conversely with such a modest gaseous $\mathrm{H} / \mathrm{He}$ fraction $(\$ 10 \%)$, the evolution of the planet is largely dominated by the core contribution. Neglecting the temperature-dependence of the core, and thus its contribution to the gravothermal energy of the planet, yields an incorrect cooling sequence. Even though, for such low-entropy material, the global gravothermal energy, $T \mathrm{~d} S / \mathrm{d} t$, remains small, a proper calculation should take into consideration the core contribution. Note also that even a modest $\mathrm{H} / \mathrm{He}$ fraction affects the radius determination, as illustrated in Table 3 by comparing the present results with the value of the radius corresponding to a pure 22.6 $M_{\oplus}$ icy planet with no gas envelope, as derived from the fitting formulae of Seager et al. (2007).

Importantly enough, taking into account the thermal and gravitational energetic contributions from the core becomes even more crucial if the irradiation effects from the parent star are important. Figure 9 shows the evolution of the same planet model,
Table 3. Radius of a $22.6 M_{\oplus}$ planet at 2 Gyr with different water core masses, EOS and levels of irradiation, characterised by a stellar incident flux, $F_{\text {inc }}$. Two cases are considered: no irradiation $\left(F_{\text {inc }}=0\right)$ and $F_{\text {inc }}=$ $6 \times F_{\text {inc }}$ of GJ 436b. Comparison is done with the pure water (ice) case of Seager et al. (2007). Results based on the assumption of a uniform core temperature of $T=300 \mathrm{~K}$ are also given.

\begin{tabular}{llll}
\hline \hline $\begin{array}{l}M_{\text {core }} \\
\left(M_{\oplus}\right)\end{array}$ & $F_{\text {inc }}$ & EOS (core) & $\begin{array}{l}R_{\mathrm{p}} \\
\left(R_{\mathrm{J}}\right)\end{array}$ \\
\hline 21 & 0 & aneos & 0.396 \\
21 & 0 & aneos $T=300 \mathrm{~K}$ & 0.379 \\
21 & 0 & sesame & 0.381 \\
21 & 0 & sesame $T=300 \mathrm{~K}$ & 0.358 \\
22.6 & 0 & Seager & 0.285 \\
21 & $6 \times F_{\text {inc }}^{\mathrm{GJ} 436}$ & aneos & 0.452 \\
21 & $6 \times F_{\text {inc }}^{\mathrm{GJ} 436}$ & aneos $T=300 \mathrm{~K}$ & 0.421 \\
21 & $6 \times F_{\text {inc }}^{\mathrm{GJ} 436}$ & sesame & 0.446 \\
21 & $6 \times F_{\text {inc }}^{\mathrm{GJ} 436}$ & sesame $T=300 \mathrm{~K}$ & 0.397 \\
\hline
\end{tabular}

with $M_{\text {core }}=21 M_{\oplus}$ of water (SESAME EOS), but with an incident stellar flux $F_{\text {inc }}=\frac{1}{2}\left(\frac{R_{\star}}{a}\right)^{2} F_{\star}$, where $F_{\star}$ is the flux from the parent star, six times larger than for GJ 436a. This corresponds to a parent star about $50 \%$ hotter. In that case, neglecting the temperature dependence of the EOS in the core and its contribution to the planet's cooling yields a $\sim 11 \%$ (7\%) smaller radius with SESAME (ANEOS) EOS (dash-dot vs long-dash curves). This stems from the larger planet interior temperature and entropy in the irradiated sequence compared with the non-irradiated one. In the irradiated case, the core temperature ranges from 5000 to $\sim 2 \times 10^{4} \mathrm{~K}$ (from the bottom of the $\mathrm{H} / \mathrm{He}$ envelope to the center), to be compared with 3000 to $\sim 10^{4} \mathrm{~K}$ in the non-irradiated case, characteristic of the temperatures expected in the rocky/icy part of Neptune and Uranus (Guillot 2005).

\section{Evolution of super Jupiter planets: Hat-P-2b and deuterium burning planets}

The final part of our study is devoted to "super-Jupiter" extrasolar planets, with masses $M_{\mathrm{P}} \gg 1 M_{\mathrm{J}}$. It is motivated by the growing number of discoveries of massive extra-solar planets, in the mass regime overlapping the one of low-mass brown dwarfs, issued from a different formation mechanism. These discoveries feed the heated debate concerning the definition of a planet and the possibility to distinguish planets from brown dwarfs of similar mass. In this context, one of the most remarkable discoveries is the transiting super-Jupiter object HAT-P-2b (also named HD 147506b), with a mass $M_{\mathrm{p}}=9.04 M_{\mathrm{J}}$ and a radius $R_{\mathrm{p}}=0.982 R_{\mathrm{J}}$ (Bakos et al. 2007). Loeillet et al. (2007) reanalysed the orbital parameters of the system and find similar values, $M_{\mathrm{p}}=8.64 M_{\mathrm{J}}, R_{\mathrm{p}}=0.952 R_{\mathrm{J}}$. In that case, the massradius relationship offers a unique information to infer the gross composition of the object and to determine its real nature, lowmass gaseous brown dwarf or very metal-rich massive planet. Bakos et al. (2007) suggest that the mean density of this planet is only marginally consistent with model predictions for an object composed predominantly of $\mathrm{H}$ and $\mathrm{He}$, and requires the presence of a large core, with $M_{\text {core }} \gtrsim 100 M_{\oplus}$. Here, we calculate more thoroughly the internal structures consistent with the radius determination, along the lines described in the previous sections. We find that a total amount of 300-600 (200-500) $M_{\oplus}$ of a water (rock) component is required to explain the radius at 


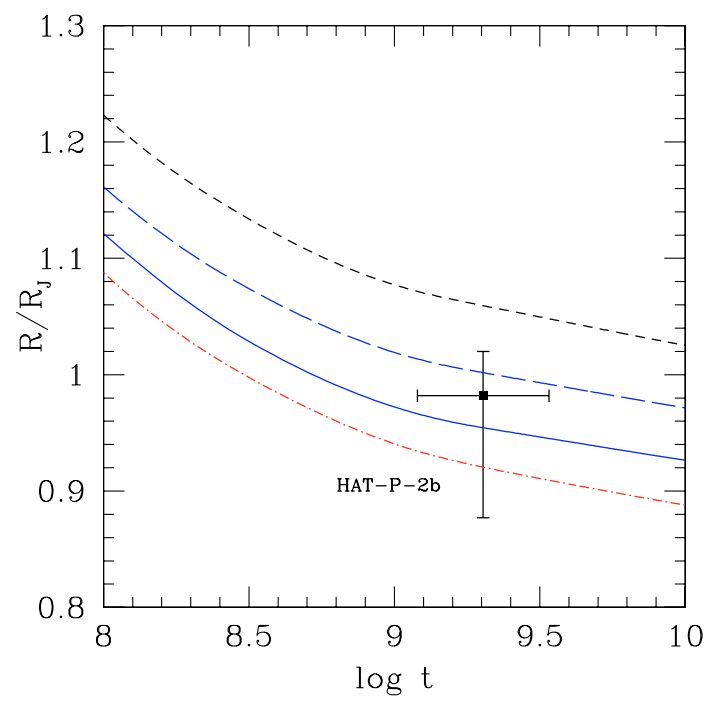

Fig. 11. Evolution of a super-Jupiter planet with the characteristics of HAT-P-2b $\left(M_{\mathrm{p}}=9 M_{\mathrm{J}}\right)$ for different distributions of heavy elements. Irradiation effects from the parent star are taken into account, adopting a time-average orbital distance $a=0.077 \mathrm{AU}$, for the proper eccentricity $e=0.52$ and semimajor axis of the relative orbit $a_{\text {rel }}=0.0677$ AU. Solid line: $M_{\text {core }}=600 M_{\oplus}$ of water (ANEOS EOS) and $Z_{\text {env }}=0$. Longdashed line: $M_{\text {core }}=350 M_{\oplus}$ of water (ANEOS EOS) and $Z_{\text {env }}=0$. Dash-dotted line: $600 M_{\oplus}$ of heavy elements with $100 M_{\oplus}$ in the core (water, with ANEOS EOS) and $500 M_{\oplus}$ in the envelope, i.e. $Z_{\mathrm{env}}=0.18$, mimicked by an $Y_{\text {equiv }}=0.275+0.18$. Short-dashed line: $\mathrm{H} / \mathrm{He}$ brown dwarf with $Z=Z_{\odot}$.

the present age, as illustrated in Fig. 11. The rather large predicted range of heavy material enrichment stems from the large observational error bars. As seen in the figure, a brown dwarf (Baraffe et al. 2003), i.e. a gaseous $\mathrm{H} / \mathrm{He}$ object with solar metallicity, is predicted to have at this age a radius marginally consistent at the $2 \sigma$ limit with the observations (short-dashed line) and thus can be excluded at the $\gtrsim 95 \%$ confidence level, if the present radius observational determination is confirmed. This shows that planets can exist with masses up to $\sim 10 M_{\mathrm{J}}$, well above the opacity-limit for fragmentation, $m \sim 3-5 M_{\mathrm{J}}$ (Whitworth \& Stamatellos 2006), the expected minimum mass for brown dwarf formation. These two distinct astrophysical populations should then overlap over a substantial mass domain.

In this context, it is interesting to explore the fate of planets massive enough to ignite deuterium-fusion in their central parts, i.e. with $M_{\mathrm{p}} \gtrsim 12 M_{\mathrm{J}}$ (Saumon et al. 1996; Chabrier et al. 2000). Indeed, recent calculations of planetary population synthesis based on improved core-accretion models of planet formation (Alibert et al. 2005a) and a wide variety of initial conditions, predict the formation of super-massive planets, up to $\sim 20-25 M_{\mathrm{J}}$, with rocky/icy core masses up to several $100 M_{\oplus}$ (Ida \& Lin 2004; Mordasini et al. 2007, 2008). If planets with a massive core can form above the aforementioned deuterium burning minimum mass, a key question is to determine whether or not the presence of the core can prevent deuterium burning to occur in the deepest layers of the $\mathrm{H} / \mathrm{He}$ envelope. Guided by the results of Mordasini et al. (2008), we have considered a $25 M_{\mathrm{J}}$ planet with a $100 M_{\oplus}$ core. Independently of the composition of the core material (water or rock), deuterium-fusion ignition does occur in the layers above the core and deuterium is completely depleted in the convective $\mathrm{H} / \mathrm{He}$ envelope after $\sim 10 \mathrm{Myr}$. The same conclusion holds for a core mass of several $100 M_{\oplus}$. These results highlight the utter confusion provided by a definition of a planet based on the deuterium-burning limit.

\section{Planet evolutionary models and mass-radius relationships}

\subsection{The reality test}

Some of the internal structures determined in the previous sections in order to match the observed radii and inferred mean densities of transit planets are rather unusual, and the possibility to form such compositions must be examined in the context of our current understanding of planet formation. According to current models of planet formation, which include migration (Alibert 2005a, 2006), up to $\sim 30 \%$ of the heavy material contained in the protoplanetary disk can be incorporated into forming giant planets (Mordasini et al. 2007). The maximum mass for a stable protoplanetary disk is $M_{D} \lesssim 0.1 M_{\star}$, so that, for a $1 M_{\odot}$ parent star of solar composition, $Z \simeq 2 \%$, as much as $M_{Z} \approx 0.3 \times 0.1 \times 0.02 \times\left(3.3 \times 10^{5}\right) \approx 200 M_{\oplus}$ of heavy material can be accreted onto the planet. Present planet formation models (Mordasini et al. 2007) reach about this limit for very massive planets, for a $1 M_{\odot}$ parent star. Therefore, in principle, according to these calculations, a heavy element mass fraction $>50 \%$ can not be excluded, even for Jovian-type planets. This requires, however, accretion rates from the planet's feeding zone significantly larger than the values typical of the early "runaway growth" accretion phase, $\sim 10^{-5} M_{\oplus} \mathrm{yr}^{-1}$ (Ward 1996). It also implies migration of the planet's embryo, or some substantial orbit eccentricity. Indeed, in the absence of migration or eccentricity, tidal interactions between the planet and the disk are supposed to lead to the opening of a gap once the planet has reached about a Saturn total (gas+solid) mass, $\sim 100 M_{\oplus}$, for the minimum mass solar nebulae conditions, after which planetesimal accretion decreases dramatically (Lin \& Papaloizou 1986). Note that, if the planets formed originally at large orbital distances and migrated inwards, they are expected to have a significant content of heavy material, given the larger available mass reservoir. Furthermore, short-period planets are expected to have a larger fraction of heavy material than planets located further away for two reasons. First of all, more impacts from low eccentricity orbit planetesimals are expected. Second of all, the closer the orbit of the planet, the larger its orbital speed, $\left(G M_{\star} / a\right)^{1 / 2}$, compared to its escape velocity, $\left(2 G M_{\star} / R_{\star}\right)^{1 / 2}$, making planetesimal ejection less efficient (Guillot 2005).

A second concern is the possibility to have the type of heavy element distributions examined in the present paper. As mentioned earlier, the fact that gas accretion is triggered once the core mass has reached about 6 to $10 M_{\oplus}$ seems to be a rather robust result (Mizuno 1980; Stevenson 1982; Pollack et al. 1996; Alibert et al. 2005a; Rafikov 2006). For larger enrichments, the rest of heavy elements should thus be mixed with the $\mathrm{H} / \mathrm{He}$ envelope. As shown by Stevenson (1982), the maximum amount of heavy material (compared with the $\mathrm{H} / \mathrm{He}$ medium) which can be redistributed uniformly by convection throughout the planet from an initially stably stratified configuration is of the order of the planet's mass. So in principle, a planet with no or small core but all or most of the heavy material being redistributed throughout the gaseous envelope is possible. As shown in our study, for Jovian type planets (see Sect. 4.2), for a global metal fraction $Z=50 \%$, if we assume a core of $10 M_{\oplus}$, comparable to what is expected in Jupiter or Saturn, and we distribute the rest in the envelope, we find essentially the same evolution as when the heavy elements are distributed throughout the whole planet, with no core. This supposes, of course, that large-scale convection remains efficient enough to redistribute homogeneously the heavy material in the $\mathrm{H} / \mathrm{He}$ envelope (Chabrier \& Baraffe 2007). Note 
that this result also holds for lower mass planets with similar enrichment, $Z=50 \%$, if the core mass remains $\$ 10 \% M_{\mathrm{p}}$.

Therefore, the enrichment in heavy material and the internal compositions explored in the present calculations have at least some reasonable theoretical foundation and can not be excluded a priori. These arguments can be examined for the case of Hat-P-2b, for which the observed mass-radius relation requires a total mass of heavy elements of at least 200 to $300 M_{\oplus}$, i.e. a mean mass-fraction $Z \gtrsim 7-11 \%$. Hat-P-2b's parent star is a F8 metal-enriched $([\mathrm{Fe} / \mathrm{H}]=0.1)$ star, with $M_{\star}=1.3 M_{\odot}$. The maximum total amount of heavy element material available in the parent disk was thus about $900 M_{\oplus}$. So the required content of heavy elements would be close to the aforementioned $\sim 30 \%$ upper limit of accretion efficiency. If both the mass and the density of Hat-P-2b are confirmed, this object thus lies at the edge of what is predicted to be possible within the current standard core-accretion scenario. We suggest, however, an alternative formation scenario, namely that the formation of Hat-P-2b involves collision(s) with one or several other massive planets. Besides forming big cores, collisions will lead to a substantial loss of the gaseous envelope, thus to a larger relative fraction of heavy elements. Furthermore, gravitational scattering among planets generally results in a tight orbit with a large eccentricity for one of the planets, which could explain Hat-P-2b's large eccentricity, and to the ejection of the other planet(s) or debris to interstellar medium. Such scattering processes between planets seem to provide a viable and possibly dominant scenario to explain the observed eccentricity distribution of exoplanets (Chatterjee et al. 2007). We thus speculate that Hat-P-2b was formed from such collision processes. Note that a scenario based on giant impacts has also been suggested to explain the large heavy element content of HD 149026b (Ikoma et al. 2006).

Finally, in the present calculations, the heavy material is supposed to be composed entirely of one single component, water, rock (silicates) or iron. This is of course a simplifying assumption, as the inner composition of the planets is expected to possess various fractions of each of these components. The water to silicate fraction, in particular, will vary depending whether the object has formed inside or beyond the ice line. Migration, however, will affect this fraction, as the migration process yields a larger collision rate of the planet embryo with rocky planetesimals and thus a decreasing abundance of volatiles. All ratios $M_{\text {ice }} / M_{\text {rock }}$ from 0 to 1 are thus probably possible. In any event, although the exact composition of the heavy material component may have some implication on the mass-radius relationship for Earth-like planets (Valencia et al. 2006; Sotin et al. 2007; Seager et al. 2007), the present study shows that, for planets with a gaseous $\mathrm{H} / \mathrm{He}$ envelope of mass fraction $M_{\mathrm{HHe}} \gtrsim 10 \% M_{\mathrm{p}}$, current uncertainties on the EOS and the heavy element distribution lead to larger uncertainties on the planet's radius determination than the effect due to different internal compositions (see Tables 1, 2 and Sect. 5.1).

\subsection{The models}

In Tables $4,5^{3}$, we present a subset of our grid of planetary models from $20 M_{\oplus}$ to $1 M_{\mathrm{J}}$ with different levels of heavy element enrichment, $Z=Z_{\odot}, 10 \%, 50 \%$ and $90 \%$. For the purpose of the present paper, this grid is restricted for the moment to solar metallicity atmosphere models with two external atmospheric conditions, namely: (i) no irradiation (non-irradiated planets)

\footnotetext{
3 The complete grid of models, from $10 M_{\oplus}$ to $10 M_{\mathrm{J}}$, is available on http: //perso. ens-lyon. fr/isabelle. baraffe/PLANET08
}

Table 4. Radii of planets (in $R_{\mathrm{J}}$ ) in the mass range $20 M_{\oplus}-1 M_{\mathrm{J}}$ for different levels of heavy element enrichment $Z$ (see text, Sect. 7.2, for the choice of the heavy material distribution) and at different ages.

\begin{tabular}{ccccc}
\hline \hline$Z$ & $M_{\mathrm{p}} / M_{\oplus}$ & $R_{0.5 \mathrm{Gyr}}$ & $R_{1 \mathrm{Gyr}}$ & $R_{5 \mathrm{Gyr}}$ \\
\hline 0.02 & 10. & 0.828 & 0.811 & 0.758 \\
& 20. & 0.858 & 0.839 & 0.800 \\
& 50. & 0.923 & 0.905 & 0.876 \\
& 100. & 0.980 & 0.963 & 0.937 \\
& 159. & 1.017 & 0.995 & 0.968 \\
0.10 & 318. & 1.057 & 1.032 & 0.998 \\
& 10. & 0.779 & 0.763 & 0.716 \\
& 20. & 0.813 & 0.797 & 0.762 \\
& 50. & 0.878 & 0.862 & 0.836 \\
& 100. & 0.935 & 0.919 & 0.896 \\
& 159. & 0.971 & 0.951 & 0.926 \\
0.50 & 318. & 1.012 & 0.990 & 0.958 \\
& 10. & 0.598 & 0.586 & 0.555 \\
& 20. & 0.632 & 0.621 & 0.598 \\
& 50. & 0.683 & 0.665 & 0.635 \\
& 100. & 0.717 & 0.697 & 0.670 \\
& 159. & 0.739 & 0.718 & 0.690 \\
0.90 & 318. & 0.781 & 0.756 & 0.721 \\
& 10. & 0.382 & 0.375 & 0.357 \\
& 20. & 0.420 & 0.414 & 0.403 \\
& 50. & 0.503 & 0.474 & 0.439 \\
& 100. & 0.543 & 0.512 & 0.469 \\
& 159. & 0.568 & 0.538 & 0.492 \\
& 318. & 0.607 & 0.578 & 0.524 \\
\hline & & & &
\end{tabular}

and (ii) irradiation effects from a Sun at $0.045 \mathrm{AU}$, which is a typical incident irradiation for most of the transit planets discovered up to now. The effect of different levels of irradiation and different atmospheric compositions will be explored in a forthcoming paper. We have compared our models in this mass range with the models of Fortney et al. (2007). We find an excellent agreement for jovian-mass planets using the same assumptions, i.e. same core masses and level of irradiation. Small differences occur for Neptune-mass planets (Fortney's $17 M_{\oplus}$ model) for a large core mass fraction $\left(M_{\text {core }}=10 M_{\oplus}\right)$. This is due most likely to the zero-temperature EOS assumption adopted in Fortney et al. (2007) for the core EOS, as shown earlier in this paper (see Sect. 4.1). Larger discrepancies, however, appear in the irradiated case for the Neptune-mass planet. This stems very likely from the different treatments of irradiation in the atmosphere models used in Forney et al. (2007; see Fortney \& Marley 2007) and in the ones used presently (Barman et al. 2001), which seem to affect more drastically light planets. The exploration of these effects will be considered in a future paper.

In the present grid of models, and in order to minimize the number of possibilities, the heavy element material is restricted to water using the SESAME EOS. The departures from this case due to different heavy material compositions (rock, iron), distributions within the planet's interior and EOS have been quantified in the previous sections of this paper (see Tables 1, 2 for an illustration and Sect. 8 for a summary) for the relevant planetmass range. The model users are thus referred to these analysis to determine the variations expected fom these various assumptions in the planet modelling. Our choice for the heavy element distribution in the model grid depends on the planet mass, and has been guided by the most realistic expected distribution, as discussed in Sect. 7.1 and summarized below, from our present analysis of the uncertainties resulting from different treatments of heavy element enrichment. The solar metallicity models, with $Z=0.02$, have the same composition as that of a brown dwarf, 
Table 5. Same as Table 4 for irradiated models by a Sun at 0.045 AU.

\begin{tabular}{ccccc}
\hline \hline$Z$ & $M_{\mathrm{p}} / M_{\oplus}$ & $R_{0.5 \mathrm{Gyr}}$ & $R_{1 \mathrm{Gyr}}$ & $R_{5 \mathrm{Gyr}}$ \\
\hline 0.02 & 10. & - & - & - \\
& 20. & 1.441 & 1.391 & 1.229 \\
& 50. & 1.258 & 1.192 & 1.084 \\
& 100. & 1.201 & 1.155 & 1.074 \\
& 159. & 1.186 & 1.151 & 1.085 \\
0.10 & 318. & 1.160 & 1.137 & 1.089 \\
& 10. & 1.517 & 1.506 & 1.428 \\
& 20. & 1.416 & 1.326 & 1.147 \\
& 50. & 1.173 & 1.120 & 1.021 \\
& 100. & 1.131 & 1.091 & 1.020 \\
& 159. & 1.120 & 1.089 & 1.032 \\
0.50 & 318. & 1.106 & 1.086 & 1.042 \\
& 10. & 1.095 & 1.050 & 0.927 \\
& 20. & 0.935 & 0.890 & 0.800 \\
& 50. & 0.841 & 0.798 & 0.725 \\
& 100. & 0.812 & 0.787 & 0.737 \\
& 159. & 0.813 & 0.790 & 0.747 \\
0.90 & 318. & 0.830 & 0.810 & 0.772 \\
& 10. & 0.545 & 0.528 & 0.492 \\
& 20. & 0.517 & 0.502 & 0.474 \\
& 50. & 0.575 & 0.534 & 0.469 \\
& 100. & 0.594 & 0.560 & 0.498 \\
& 159. & 0.601 & 0.574 & 0.522 \\
& 318. & 0.628 & 0.604 & 0.552 \\
\hline \multirow{6}{*}{0.}
\end{tabular}

but such objects with masses as low as a few Neptune-masses are of course not realistic. These models are an extension to planetary masses of the models of Baraffe et al. (2003). Note that the $20 M_{\oplus}$ planet model with an enrichment as low a $Z=0.02$ and with, consequently, a low mean density, expands under the effect of irradiation. This model is not included in Table 5, being meaningless.

- For all masses and $Z=10 \%$, all heavy elements are located in the core, since for such low $Z$, we have shown that their distribution has only a modest effect on the radius.

- For larger enrichments $(Z=50 \%-90 \%)$, we make a distinction between planets below $M_{\mathrm{p}} \lesssim 20 M_{\oplus}$, hereafter denominated as "light planets", and more massive planets. Because all planets are expected to have a core of about $\sim 10 M_{\oplus}$, the following distributions seem to be the most realistic:

- for light planets, all heavy material is located in the core.

- for more massive planets, we have shown that distributing the heavy elements over the entire planet is similar to distributing them partly in a core of at most $\sim 10 \%$ of the mass of the planet and partly in the envelope. We thus adopt such a distribution for these objects: the heavy material is distributed over the entire planet, using the AVL with SESAME EOS. The uncertainties due to the EOS (ANEOS versus SESAME) for this type of distribution have been quantified in Sects. 4 and 5.

- for planets with masses $>1 M_{\mathrm{J}}$, we only provide models with $Z=10 \%$, since enrichments as large as $50 \%$ or $90 \%$ correspond to an amount of heavy material greater than the quantity available in protoplanetary disks around solar type stars.

Figure 12 shows the mass-radius relationships for planets in the mass range $20 M_{\oplus}-1 M_{\mathrm{J}}$, for different levels of irradiation and heavy element enrichments. The models are compared to the observed mass-radius data of transiting planets. Note that in the case of irradiation (right panel), the theoretical planetary radius is not corrected from the effect of the atmospheric extension.
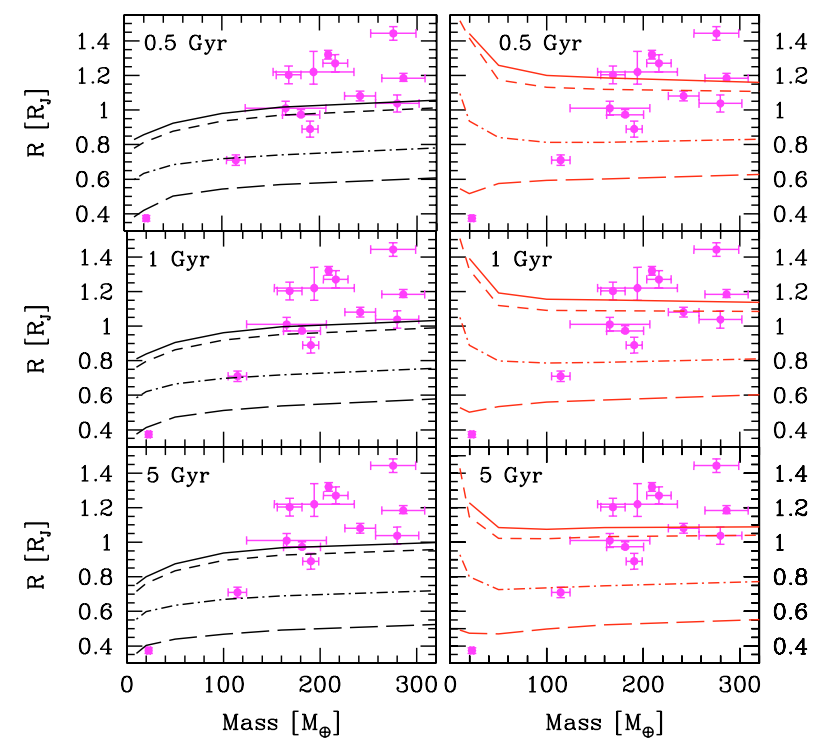

Fig. 12. Mass-radius relationships for planets in the mass range $10 M_{\oplus}-1 M_{\mathrm{J}}$ at different ages, as indicated in the panels, and different levels of heavy element enrichment: $Z=Z_{\odot}$ (solid lines); $Z=10 \%$ (short-dash lines); $Z=50 \%$ (dash-dot lines); $Z=90 \%$ (long-dash lines). Left panels: without irradiation; right panels: with irradiation from a Sun at 0.045 AU. Symbols indicate the observed data for transiting Neptune-mass to Jupiter mass planets, taken from F. Pont's website (wWw. inscience.ch/transits).

Such a correction adds $\sim 4 \%$ to the calculated radius for this level of irradiation (Baraffe et al. 2003).

\section{Discussion and perspectives}

In this paper, we have explored the uncertainties in current planetary structure and evolutionary models arising from the treatment of the heavy material component in the interior of these planets. The study covers a mass range from $10 M_{\oplus}$ to $1 M_{\mathrm{J}}$. Our main results can be summarized as follows:

- The ideal mixing entropy contribution arising from the heavy $Z$-material is found to be inconsequential on the planet's evolution, within the limit that the variation of the degree of ionization of these elements along the evolution is presently ignored. This mixing entropy, however, represents about 10 to $20 \%$ of the total, $\mathrm{H} / \mathrm{He}+Z$ elements, entropy, and thus modifies the internal isentrope. A proper calculation of the planet's structure for a given entropy should thus include this contribution.

- For a metal mass fraction in the envelope $Z_{\text {env }} \lesssim 20 \%$, the EOS of the $Z$-material can be approximated by using a corresponding $Y_{\text {equiv }}$ effective helium mass fraction in the SCVH EOS. Above this limit, this approximation becomes more and more incorrect and yields erroneous cooling sequences.

- For core mass fractions less than $50 \%$ of the planet's mass, a variation of the core composition between pure water and pure rock (iron) yields a difference on the radius of less than $7 \%(15 \%)$ after $1 \mathrm{Gyr}$, for all the planet masses of interest.

- For a total mass fraction of heavy elements $Z \lesssim 10 \%-15 \%$, their impact on the evolution of the planet can be mimicked reasonably well by assuming that they are all located in the core.

- For heavy material enrichments $Z>20 \%$, the distribution of heavy elements (everything in the core versus uniform distribution) can affect significantly the cooling and thus 
the radius determination (more than $10 \%$ at a given age). Therefore:

- For metal-rich $(Z \gtrsim 20 \%)$ light planets $\left(\$ 20 M_{\oplus}\right)$, since the planets are expected to have a massive $\sim 10 M_{\oplus}$ core, it seems realistic to put all the heavy material in the core.

- For massive metal-rich planets $\left(M_{\mathrm{p}} \gtrsim 50 M_{\oplus}\right.$ and $\left.Z>20 \%\right)$, however, the evolution is better described by models which assume that the heavy elements are distributed throughout the entire planet than by models with all heavy elements in the core and none in the gaseous envelope. The former models yield results similar to the ones obtained with a more realistic distribution, namely a $\sim 10 M_{\oplus}$ core and the rest of heavy material distributed in the envelope.

- The temperature dependence of the heavy material EOS and the release of gravitational and thermal energy of the core have negligible effects on the cooling history of massive planets (saturnian and jovian masses), independently of the core mass. For Neptune mass planets, these effects are significant ( $\sim 10 \%$ difference on the radius after $1 \mathrm{Gyr})$ for extreme heavy element enrichments $(Z>90 \%)$, as for instance in GJ 436b.

We have performed comparisons between available EOS of heavy material which cover a range of temperature and pressure large enough to cover the characteristic domains of exoplanet interiors. Unfortunately, significant differences exist between these EOS concerning the thermodynamical quantities relevant for planet evolution, such as the entropy, in particular at high pressures $(P \gg 0.01 \mathrm{Mbar})$ and temperatures $(T \gg 5000 \mathrm{~K})$, where no experimental or numerical guidance is provided. These discrepancies translate into non-negligible variations on the massradius relation. Therefore, by venturing into parts of the phase diagram of heavy elements that are presently not possible to reach experimentally or with computer numerical simulations, the planet internal structure calculations include inevitably a certain degree of uncertainty, which has been quantified as thoroughly as possible in the present study. For planets with even a modest fraction of $\mathrm{H} / \mathrm{He}$ gaseous envelope, as expected above $\sim 6-10 M_{\oplus}$, these heavy material EOS related uncertainties are the major culprit for preventing accurate determinations of the exoplanet internal composition from the observed mass and radius. "Accurate" means in this context at a level better than yielding $\$ 10 \%$ variations on the radius. For Earth or super-Earth planets with no gaseous envelope, a radius measurement accuracy better than $5 \%$ is expected to allow to distinguish icy from rocky internal compositions (Valencia et al. 2007). However, as shown in Sect. 5 for the specific cases of HD 149026b and GJ 436b, in the presence of even a small $(<30 \%$ by mass $) \mathrm{H} / \mathrm{He}$ contribution, varying the distribution of the heavy material within the planet has by itself a larger impact, not mentioning the one due to the uncertainty in the EOS. Therefore, for planets above $\sim 10 M_{\oplus}$, massive enough to accrete an $\mathrm{H} / \mathrm{He}$ envelope, it seems difficult to determine precisely the internal composition with current structure models, as the effect of the heavy material composition on the radius is blurred by the presence of a gaseous envelope. Even a modest fraction $(\sim 10 \%)$ of $\mathrm{H} / \mathrm{He}$ already severely modifies the evolution and thus the radius of a planet compared with a gasless one. As an example, a $10 M_{\oplus}$ mass planet retaining a $10 \%$ $\mathrm{H} / \mathrm{He}$ envelope is $\sim 1.5$ times larger than its pure icy counterpart (Seager et al. 2007). Frustratingly enough, the impact of this gas contribution casts doubt on our ability to determine accurately these planet inner composition from the observed radius, at a better level than figuring out their gross bulk properties, already an important information for our understanding of planet formation.

The main goal of this work was to explore thoroughly current uncertainties and assumptions in current models of planet structure and evolution. Such a study had been eluded so far in the field of exoplanets and becomes now necessary, given the high accuracy achievable by present and forthcoming observations. This work enters and prolongates the long history of the modeling of giant planets, where some of the problems and uncertainties discussed in the present paper have been addressed decades ago for the case of our own Solar System planets. As an example, the sensitivity of Uranus and Neptune models to the EOS of water at high pressure has been thoroughly discussed by Hubbard \& MacFarlane (1980). The importance of the thermal and gravitationnal energy release of heavy material in planet interiors was already stressed for the evolution of Uranus and Netpune (Podolak et al. 1991; Hubbard et al. 1995). All this historical work has already highlighted the remaining large uncertainties on the modeling of our own giant planets, despite a wealth of observational constraints which are lacking for exoplanets.

Although our conclusions sound rather pessimistic, concerning the degree of accuracy expected from current planet models, this work should motivate further efforts both on the experimental and theoretical fronts, in order to make progress in this thriving field of exoplanet exploration. The (non exhaustive) homework list of improvements includes the following items. (i) Exploring the EOS of heavy material in the critical pressure regime $0.1-100 \mathrm{Mbar}$ and at high temperature is crucial. Substantial progress in this domain is expected with the ongoing and future high-pressure experiments in various national laboratories (including e.g. the Lawrence Livermore and Sandia labs in the US or the LIL and MegaJoule laser projects in France). (ii) In the same vein, first-principle $N$-body numerical methods (DFT, path-integral, quantum molecular dynamics, ...) should be able to provide at least some benchmarks in the part of the $P-T$ diagram which for now lies in the unknown interpolated regime. (iii) It is important to explore the efficiency of the heat transport mechanism in planetary interiors in the presence of molecular weight gradients, in particular in the case of large heavy material enrichment. This bears crucial consequences on the planet's cooling history (Chabrier \& Baraffe 2007). Current progress in high-resolution multi-D numerical simulations should be able to handle this problem. (iv) Even though measuring the extrasolar planet gravitational moments is hardly conceivable, it might be possible to determine their oblateness, $e=R_{\mathrm{eq}} /\left(R_{\mathrm{eq}}-R_{\mathrm{pol}}\right)$, where $R_{\mathrm{eq}}$ and $R_{\mathrm{pol}}$ denote the equatorial and polar radius, respectively, with future transit observations (Seager \& Hui 2002; Barnes \& Fortney 2003). This in turn leads to the determination of the rotation rate and thus of the centrifugal potential, providing a more stringent constraint on the internal structure of the planet. (v) Last but not least, the perspective, on the observational front, of direct planetary atmosphere observations (LYOT project, GEMINI, ELT, DARWIN/TPF or their precursors) and transit detections (CoRoT, Kepler) will improve our knowledge of their surface composition and radius measurements and provide important constraints on the planet's content in heavy material.

Finally, we have shown in this paper that massive ( $z$ several Jupiter mass) planets may form from two different avenues, namely the standard core accretion scenario (Mordasini et al. 2008), and giant impacts between massive planets or planet embryos. This latter process is likely to yield very metal-enriched and thus very dense massive planets, with a finite eccentricity, as a result of planet scattering. Objects like HD 149026b, 
Hat-P-2b or the very recently discovered HD 17156b (Barbieri et al. 2007) could be the illustrations of this latter planet formation mechanism. The observation of Hat-P-2b, together with the numerous observations of free floating brown dwarfs of a few Jupiter-masses (Caballero et al. 2007) shows that planets and brown dwarfs have a substantial (about one order of magnitude in mass) mass domain overlap. As we have shown in this paper, planets massive enough to exceed the deuterium-burning mass limit will indeed ignite this reaction at the bottom of their $\mathrm{H} / \mathrm{He}$ rich envelope, at the top of the core. This is one more evidence, if it were still necessary, that using the deuterium-burning limit as a criterion to distinguish planets from brown dwarfs has no valid foundation.

The complete grid of models, from $10 M_{\oplus}$ to $10 M_{\mathrm{J}}$, is available on http://perso.ens-lyon.fr/isabelle.baraffe/ PLANETQ8

Acknowledgements. The authors thank D. Saumon for useful discussions during the elaboration of this work and our referee, J. Fortney, for his valuable comments. Part of this work was done as I.B. and G.C. were visiting the University of Toronto and the Max-Planck Institut for Astrophysics in Garching; these authors thank these departments for their hospitality. The financial support of Programme National de Physique Stellaire (PNPS) and Programme National de Planétologie of CNRS/INSU (France) is aknowledged.

\section{Appendix A: Calculation of the mixing entropy}

In this appendix, we calculate the expression for the ideal mixing entropy of a two-component system composed of a H/He mixture, identified as one component, on one side and of a heavy material component of mass fraction $Z=M_{Z} / M$ on the other side. In the following, the subscript " 1 " denotes the H/He component while the subscript " 2 " refers to the $Z$-component. By definition, the ideal entropy of mixing reads:

$\frac{S_{\text {mix }}}{k_{\mathrm{B}}}=\mathcal{N} \ln \mathcal{N}-N_{1} \ln N_{1}-N_{2} \ln N_{2}-N_{\mathrm{e}} \ln N_{\mathrm{e}}$,

where $\mathcal{N}=\mathcal{N}_{\infty}+\mathcal{N}_{\in}$ denotes the total number of particles, including free electrons, in the fluid, $\mathcal{N}_{i}=N_{i}+N_{\mathrm{e}}^{i}$ denotes the total number of particles of component $i$, with $N_{i}$ the number of nuclei of component $i$ and $N_{\mathrm{e}}^{i}$ the number of electrons provided by the component $i$. Developing Eq. (A.1) yields:

$$
\begin{aligned}
\frac{S_{\text {mix }}}{k_{\mathrm{B}}}= & \mathcal{N}_{1} \ln \left(1+\frac{\mathcal{N}_{2}}{\mathcal{N}_{1}}\right)+\mathcal{N}_{2} \ln \left(1+\frac{\mathcal{N}_{1}}{\mathcal{N}_{2}}\right) \\
& +\mathcal{N}_{1} \ln \mathcal{N}_{1}+\mathcal{N}_{2} \ln \mathcal{N}_{2} \\
& -N_{1} \ln N_{1}-N_{2} \ln N_{2}-N_{\mathrm{e}} \ln N_{\mathrm{e}} \\
= & \mathcal{N}_{1} \ln \left(1+\frac{\mathcal{N}_{2}}{\mathcal{N}_{1}}\right)+\mathcal{N}_{2} \ln \left(1+\frac{\mathcal{N}_{1}}{\mathcal{N}_{2}}\right) \\
& -N_{\mathrm{e}} \ln N_{\mathrm{e}}+N_{\mathrm{e}}^{1} \ln N_{\mathrm{e}}^{1} \\
& +N_{\mathrm{e}}^{2} \ln N_{\mathrm{e}}^{2}+\frac{S_{\text {mix }}^{(1)}}{k_{\mathrm{B}}}+\frac{S_{\text {mix }}^{(2)}}{k_{\mathrm{B}}}
\end{aligned}
$$

where

$$
\frac{S_{\mathrm{mix}}^{(i)}}{k_{\mathrm{B}}}=\mathcal{N}_{\mathrm{i}} \ln \mathcal{N}_{\mathrm{i}}-N_{\mathrm{i}} \ln N_{\mathrm{i}}-N_{\mathrm{e}}^{\mathrm{i}} \ln N_{\mathrm{e}}^{\mathrm{i}}
$$

denotes the ideal mixing entropy of the component $i$, including various ionic, atomic or molecular species as well as electron contributions (see SCVH), and $N_{\mathrm{e}}=N_{\mathrm{e}}^{1}+N_{\mathrm{e}}^{2}$ is the total number of electrons provided by the $\mathrm{H} / \mathrm{He}$ and $Z$ components. These ideal mixing entropy contributions are already included in the $\mathrm{SCVH}$ EOS for the $\mathrm{H} / \mathrm{He}$ component (their Eq. (53) $)^{4}$ and in

\footnotetext{
4 Note the following typos in the SCVH paper. In Eqs. (45), (46), the entropy ratios on the rhs of the equations should be $\frac{S^{\mathrm{H}}}{S}$ and $\frac{S^{\mathrm{He}}}{S}$ and not $\frac{S}{S^{\mathrm{H}}}$ and $\frac{S}{S^{\mathrm{He}}}$. In Eq. (56) for the parameter $\delta$, the fraction in front of the bracketed terms on the rhs of the equation should be $\frac{2}{3}$ and not $\frac{3}{2}$.
}

the appropriate EOS for the $Z$-component. Removing these two contributions, we obtain the ideal entropy of mixing which arises only from the mixture of the $\mathrm{H} / \mathrm{He}$ and $\mathrm{Z}$ components:

$$
\begin{aligned}
\frac{S_{\text {mix }}}{k_{\mathrm{B}}}= & \mathcal{N}_{1} \ln \left(1+\frac{\mathcal{N}_{2}}{\mathcal{N}_{1}}\right)+\mathcal{N}_{2} \ln \left(1+\frac{\mathcal{N}_{1}}{\mathcal{N}_{2}}\right) \\
& -N_{\mathrm{e}} \ln N_{\mathrm{e}}+N_{\mathrm{e}}^{1} \ln N_{\mathrm{e}}^{1}+N_{\mathrm{e}}^{2} \ln N_{\mathrm{e}}^{2} .
\end{aligned}
$$

The specific entropy, i.e. the entropy per unit mass is given by

$\frac{\tilde{S}_{\text {mix }}}{k_{\mathrm{B}}}=\frac{S_{\text {mix }}}{M k_{\mathrm{B}}}=\frac{\mathcal{N}}{M} \frac{S_{\text {mix }}}{\mathcal{N} k_{\mathrm{B}}}$

where $M=M_{1}+M_{2}$ is the total mass.

We define a mean atomic mass $\bar{m}_{i}=M_{i} / N_{i}$ and mean charge $1+\bar{Z}_{i}=\mathcal{N}_{i} / N_{i}$ for each component, where we have used the electroneutrality condition $N_{\mathrm{e}}^{i}=N_{i} \bar{Z}_{i}$, so that $\frac{M_{i}}{\mathcal{N}_{i}}=\bar{m}_{i} /\left(1+\bar{Z}_{i}\right)$. For the $\mathrm{H} / \mathrm{He}$ component, the quantities $\bar{m}_{1}$ and $\bar{Z}_{1}$ are given in terms of the relative number fractions $x$ of $\mathrm{H}_{2}, \mathrm{H}, \mathrm{He}, \mathrm{He}^{+}, \mathrm{He}^{++}$ and $\mathrm{e}^{-}$by the SCVH EOS (their Eqs. (33)-(35)), with:

$\frac{M_{1}}{\mathcal{N}_{1}}=\left(2 x_{\mathrm{H}_{2}}+x_{\mathrm{H}}+x_{\mathrm{H}^{+}}\right) m_{\mathrm{H}}+\left(x_{\mathrm{He}^{2+}}+x_{\mathrm{He}^{+}}+x_{\mathrm{He}}\right) m_{\mathrm{He}}$

and

$\bar{Z}_{1}=\frac{x_{\mathrm{e}}^{(1)}}{1-x_{\mathrm{e}}^{(1)}}$

where

$x_{\mathrm{e}}^{(1)}=\frac{N_{\mathrm{e}}^{1}}{\mathcal{N}_{1}}=\frac{1}{1+\beta \gamma} x_{\mathrm{H}^{+}}+\frac{\beta \gamma}{1+\beta \gamma}\left(x_{\mathrm{He}^{+}}+2 x_{\mathrm{He}^{2+}}\right)$

denotes the number-concentration of free electrons in the $\mathrm{HHe}$ mixture as defined in SCVH, $m_{\mathrm{H}}$ and $m_{\mathrm{He}}$ denote the atomic mass of hydrogen and helium, respectively, and the grec symbols $\alpha, \beta, \gamma$ are defined by Eqs. (54)-(56) of SCVH. For the $Z$-component, the mean mass corresponds to the atomic mass of the compound under consideration. The mean charge $\bar{Z}_{2}$ is unknown. We have carried out calculations for the two limiting cases of fully neutral $\left(\bar{Z}_{2}=0\right)$ and fully ionised $\left(\bar{Z}_{2}=Z_{\text {nuc }}\right)$ heavy material, where $Z_{\text {nuc }}$ denotes the nuclear charge of the compound.

After some algebra, the ideal specific entropy of mixing of the $(\mathrm{HHe}) / \mathrm{Z}$ mixture can be written:

$$
\begin{aligned}
\frac{\tilde{S}_{\text {mix }}=}{k_{\mathrm{B}}}= & \frac{S_{\text {mix }}}{M k_{\mathrm{B}}} \\
= & \frac{X_{1}}{\bar{m}_{1}}\left(1+\bar{Z}_{1}\right) \times\left\{\ln \left(1+\beta^{\prime} \gamma^{\prime}\right)+\beta^{\prime} \gamma^{\prime} \ln \left(1+\frac{1}{\beta^{\prime} \gamma^{\prime}}\right)\right. \\
& \left.-x_{\mathrm{e}}^{(1)} \ln \left(1+\delta^{\prime}\right)-\beta^{\prime} \gamma^{\prime} x_{\mathrm{e}}^{(2)} \ln \left(1+\frac{1}{\delta^{\prime}}\right)\right\}
\end{aligned}
$$

where $X_{1}=M_{1} / M \equiv M_{\mathrm{HHe}} / M$ and

$\beta^{\prime}=\frac{\bar{m}_{1}}{\bar{m}_{2}} \frac{Z}{1-Z}, \gamma^{\prime}=\frac{1+\bar{Z}_{2}}{1+\bar{Z}_{1}}, \delta^{\prime}=\frac{N_{\mathrm{e}}^{2}}{N_{\mathrm{e}}^{1}}=\beta^{\prime} \frac{\bar{Z}_{2}}{\bar{Z}_{1}}=\frac{x_{\mathrm{e}}^{(2)}}{x_{\mathrm{e}}^{(1)}} \beta^{\prime} \gamma^{\prime}(\mathrm{A} .10)$

with $Z=M_{Z} / M$ and $x_{\mathrm{e}}^{(\mathrm{i})}=\bar{Z}_{\mathrm{i}} /\left(1+\bar{Z}_{\mathrm{i}}\right)$.

\section{References}

Adams, E. R., Seager, S., \& Elkins-Tanton, L. 2007, ApJ, in press [arXiv: astro-ph/0710.4941]

Alibert, Y., Mordasini, C., Benz, W., \& Winisdoerffer, C. 2005a, A\&A, 434, 343 Alibert, Y., Mousis, O., Mordasini, C., \& Benz, W. 2005b, ApJ, 626, L57

Alibert, Y., Baraffe, I., Benz, W., et al. 2006, A\&A, 455, L25

Bakos, G. A., Kovacs, G., Torres, G., et al. 2007, ApJ, submitted protect [arXiv: astro-ph/7050126] 
Baraffe, I, Alibert, Y, Chabrier, G., \& Benz, W. 2006, A\&A, 450, 1221 Baraffe, I., Chabrier, G., Barman, T., Allard, F., \& Hauschildt, P. 2003, A\&A, 402, 701

Barbieri, M., Alonso, R., Laughlin, G., et al. 2007, A\&A, 476, L13

Barman, T., Hauschildt, P., \& Allard, F. 2001, ApJ, 556, 885

Barnes, J. W., \& Fortney, J. 2003, ApJ, 588, 545

Bodenheimer, P., Laughlin, G., \& Lin, D. N. C. 2003, ApJ, 592, 555

Burrows, A., Hubeny, I., Budaj, J., \& Hubbard, W. B. 2007, ApJ, 661, 502

Caballero, J., et al. 2007, A\&A, 470, 903

Chabrier, G., \& Baraffe, I. 2007, ApJ, 661, L81

Chabrier, G., Saumon, D., Hubbard, W. B., \& Lunine, J. 1992, ApJ, 391, 817

Chabrier, G., Baraffe, I., Allard, F., \& Hauschildt, P. 2000, ApJ, 542, L119

Chatterjee, S., Ford, E., \& Rasio, F. 2007 [arXiv: astro-ph/0703166]

Deming, D., Harrington, J., Laughlin, G., et al. 2007, ApJ, 667, L199

Demory, B.-O., Gillon, M., Barman, T., et al. 2007, A\&A, 475, 1125

Fontaine, G., Graboske, H. C. Jr., \& Van Horn, H. M. 1977, ApJS, 35, 293

Fortney, J., \& Marley, M. S. 2007, ApJ, 666, L45

Fortney, J., Saumon, D., Marley, M., Lodders, K., \& Freedman, R. S. 2006, ApJ, 642,495

Fortney, J., Marley, M. S., \& Barnes, J. W. 2007, ApJ, 659, 1661

Gillon, M., Pont, F., Demory, B.-O., et al. 2007a, A\&A, 472, L13

Gillon, M., Demory, B.-O., Barman, T., et al. 2007b, A\&A, 472, L51

Gillon, M., et al. 2007c [arXiv:0712.2073]

Guillot, T. 2005, Ann. Rev. of Earth and Planetary Sciences, 33, 493

Guillot, T., Santos, N. C., Pont, F., et al. 2006, A\&A, 453, L21

Hubbard, W. B., \& MacFarlane, J. J. 1980, JGR, 85, 225

Hubbard, W. B., \& Marley, M. 1989, Icarus, 78, 102

Hubbard, W. B., Podolak, M., \& Stevenson, D. J. 1995, Neptune and Triton book, 109

Ida, S., \& Lin, D. N. C. 2004, ApJ, 604, 388

Ikoma, M., Guillot, T., Genda, H., Tanigawa, T., \& Ida, S. 2006, ApJ, 650, 1150

Knutson, H. A., Charbonneau, D., Allen, L., et al. 2007, Nature, 447, 183

Lin, D., \& Papaloizou, J. 1986, ApJ, 309, 846

Lin, J.-F., Gregoryanz, E., Struzhkin, V., et al. 2005, Geoph. Res. Lett., 32, 11306

Linden, P. F., \& Shirtclife, T. G. 1978, J. Fluid Mech., 87, 417
Loeillet, B., Shporer, A., Bouchy, F., et al. 2007, A\&A, submitted [arXiv: 0707.0679]

Lyon, S. P., \& Johnson, J. D. 1992, LANL Rep. LA-UR-92-3407 (Los Alamos: LANL)

Marley, M., Fortney, J., Seager, S., \& Barman, T. 2007, in Protostars and Planets V, ed. B. Reipurth, D. Jewitt, \& K. Keil (University of Arizona Press), 733

Mizuno, Z. 1980, Prog. Th. Phys., 64, 544

Mordasini, C., Alibert, Y., \& Benz, W. 2007, in Extreme Solar Systems, ed. D. Fischer, F. Rasio, S. Thorsett, \& A. Wolszczan, Santorini, ASP Conf. Ser.

Mordasini, C., Alibert, Y., Benz, W., \& Naef, D. 2008, A\&A, in preparation

Podolak, M., Hubbard, W. B., \& Stevenson, D. J. 1991 (Uranus, Tucson, AZ: University of Arizona Press), 29

Pollack, J. B., James, B., Hubickyj, O., et al. 1996, Icarus, 124, 62

Potekhin, A. 1999, A\&A, 351, 787

Rafikov, R. 2006, ApJ, 648, 666

Sato, B., Fischer, D. A., Henry, G. W., et al. 2005, ApJ, 633, 465

Saumon, D., \& Guillot, T. 2004, ApJ, 609, 1170

Saumon, D., Chabrier, G., \& VanHorn, H. M. 1995, ApJS, 99, 713

Saumon, D., Hubbard, W. B., Burrows, A., et al. 1996, ApJ, 460, 993

Seager, S., Kuchner, M., Hier-Majumder, C. A., \& Militzer, B. 2007, ApJ, 669, 1279

Seager, S., \& Hui, L. 2002, ApJ, 574, 1004

Selsis, F., Chazelas, B., Bordé, P., et al. 2007, Icarus, 191, 453

Schwegler, E., Galli, G., Gygi, F., \& Hood, R. 2001, Phys. Rev. Lett., 87, 265501

Stevenson, D. J. 1982, Ann. Rev. of earth and planetary sc., 10, 257

Stevenson, D. J. 1985, Icarus, 62, 4

Sotin, C., Grasset, O., \& Mocquet, A. 2007, Icarus, 191, 337

Thompson, S. L., \& Lauson, H. S. 1972, Improvements in the chart D Radiationhydrodynamics CODE III: revised analytic equation of state, Technical Report SC-RR-61 0714, Sandia National Laboratories

Valencia, D., O'Connell, R. J., \& Sasselov, D. 2006, Icarus, 181, 545

Valencia, D., Sasselov, D. D., \& O'Connell, R. J. 2007, ApJ, 665, 1413

Ward, W. 1996, in Completing the Inventory of the Solar System, ed. T. W. Rettig, \& J. M. Hahn, ASPC Proc., 107, 337

Whitworth, A., \& Stamatellos, D. 2006, A\&A, 458, 817 